\title{
Perturbations of quadratic Hamiltonian systems with symmetry 1
}

by

\section{Emil Ivanov HOROZOV}

University of Sofia, Faculty of Mathematics and Informatics, 5, J. Bourchier blvd., 1126 Sofia, Bulgaria.

and

\section{Iliya Dimov ILIEV}

Institute of Mathematics, Bulgarian Academy of Sciences, P.O. Box 373, 1090 Sofia, Bulgaria.

ABSTRACT. - It is proved that arbitrary quadratic perturbations of quadratic Hamiltonian systems in the plane possessing central symmetry can produce at most two limit cycles.

Key words: Bifurcation, limit cycle, quadratic vector field, Abelian integrals.

RÉSUMÉ. - Nous prouvons que les perturbations quadratiques arbitraires des systèmes hamiltoniens quadratiques et symétrie sur la plane peut produire au plus deux cycles limites.

\section{O. INTRODUCTION}

In this paper we prove the following result:

\footnotetext{
1 Research partially supported by Grants MM - 402/94 and MM - 410/94 of the NSF of Bulgaria. 
THEOREM 1. - Let $H$ be a generic cubic Hamiltonian with a central symmetry: $H(-x,-y)=-H(x, y)$. Then any quadratic perturbation of the corresponding Hamiltonian system

$$
\mid \begin{aligned}
& \dot{x}=H_{y}+\varepsilon f(x, y) \\
& \dot{y}=-H_{x}+\varepsilon g(x, y)
\end{aligned}
$$

has at most two limit cycles for $\varepsilon$ small enough.

Our interest in systems (0.1) was motivated by the project to obtain an explicit and sharp bound $c(2)$ for the number of limit cycles in quadratic vector fields which are close to Hamiltonian ones or at least to obtain an explicit sharp bound for the so called Hilbert-Arnold problem [2] for $n=2$ (see [9], [12], [15], [16], [27] for results in this direction). The HilbertArnold problem is formulated as follows: find an upper bound $Z(n)$ for the number of isolated zeros of the integral

$$
I(h)=\int_{\delta(h)}-f d y+g d x, \quad h \in \Delta
$$

where $\delta(h)$ is the oval (compact connected component) of the algebraic curve $H(x, y)=h$ and $\operatorname{deg} H=n+1, \operatorname{deg} f, g=n$ (or more generally, find an upper bound $Z(n, m)$ provided $\max (\operatorname{deg} f, \operatorname{deg} g)=m$ ).

Integrals of this type in studies of limit cycles were introduced by Pontrjagin [23]. The first nontrivial case was considered by Bogdanov [6]. The existence of an upper bound $Z(n)$ was established by A. Varchenko [25] and Khovanskii [20]. But making this bound explicit seems to be a difficult problem. Quite recently, Yu. Il'yashenko and S. Yakovenko [19] proved a double exponential estimate for $Z(n, m)$ provided $H$ is a fixed Hamiltonian from some dense set of generic Hamiltonians: $Z(n, m) \leq 2^{2^{O(m)}}$. Although this bound is far from the expected (polynomial) one, up to now this is the best known result.

More generally, one can consider perturbations of integrable systems instead of Hamiltonian ones. In the quadratic case, an essential part of the problem also consists (see [28]) in counting the isolated zeros of certain (not necessarily Abelian) integrals. Żolądek has proved [28] that in quadratic perturbations of Lotka-Volterra systems with a center the corresponding integrals will have at most two zeros. Among the remaining three classes of quadratic systems with a center, most of results have been obtained for the Hamiltonian case (see e.g. [17]). Almost nothing is known about the other two classes, where at least three limit cycles can appear. 
In a recent paper [16] we found that for generic cubic Hamiltonians with three saddles and one centre the exact value for both $c(2)$ and $Z(2)$ is two. For the two remaining basic classes of cubic Hamiltonians (namely those with one saddle and one centre and respectively with both two saddles and centres) the problem is still open. Our conjecture is that the exact bound for all generic cases is two (the same bound was conjectured in [28]). The symmetric Hamiltonians form a co-dimension one subset within the class of Hamiltonians having two saddles and two centres. For many reasons it seems to us that the symmetric case is the one allowing to be treated comparatively easier at least as far as the computations are concerned.

In the statement of Theorem 1 as well as in the above comments we use the notion of "generic" Hamiltonian. There are several definitions of this notion even for quadratic Hamiltonian vector fields. Throughout this paper we say the cubic $H$ is generic if the corresponding Hamiltonian vector field $d H=0$ has a centre and does not belong simultaneously to any of the other integrable classes of quadratic vector fields (as listed in [28]). The latter condition has a simple geometrical interpretation: nongeneric are exactly those Hamiltonians for which the level curves in suitable coordinates have an axis of symmetry. On its hand evidently the central symmetry of $H$ geometrically causes $d H=0$ to be centrosymmetric with respect to a suitable point $\left(x_{0}, y_{0}\right)$, which we without loss of generality have chosen to coincide with the origin. The general case $H\left(x_{0}-x, y_{0}-y\right)=-H\left(x-x_{0}, y-y_{0}\right)$ is dealt with a mere translation.

One can easily check that after eventual linear change of the variables each cubic Hamiltonian with a central symmetry and having a centre can be written in the form

$$
H=x y^{2}+\frac{x^{3}}{3}+\mu y-x, \quad \mu \in[0,1) .
$$

Then "generic" in this case means $\mu \neq 0$. Selected level curves of $H$ for $\mu=0, \mu \in(0,1)$ and $\mu=1$ are drawn in Fig. 1 .

System (0.1) has attracted the interest of several other authors [4], [9], [21]. In particular Bamón [4] found a value of $\mu\left(\mu=\left(4^{\frac{1}{3}}-1\right) /\left(4^{\frac{1}{3}}+1\right)=\right.$ $0,227 \ldots$ ) and a suitable perturbation for which around one of the foci a saddle loop and at least one limit cycle can coexist. Drachman et al. [9] proved that for $\mu$ close to 1 and for a specific perturbation $(f, g)$ the system $(0.1)$ has two limit cycles around one of the foci and has no limit cycle around the other one. (In [4], [9] a different coordinate system was used). Our paper covers all generic symmetric Hamiltonians and all small quadratic perturbations showing that the sharp estimate of the total number of limit cycles in (0.1) is two. Moreover from our analysis it follows that 
all numbers $\leq 2$ and positions of the limit cycles permitted by the general theory of quadratic systems [7] can be realized. It is also easy to describe all bifurcations when the parameters of $(f, g)$ vary. Some of these results (for special perturbations) were announced in [21] but apparently without proofs.

In this paper we do not consider the degenerate case $\mu=0$ which requires an analysis up to a fourth order in $\varepsilon$. This case as well as the other (nonsymmetric) degenerate cases are an object of another research (in progress).

Perhaps it is worth noticing that system (0.1) falls into class III $_{a \neq 0}$ of the Chinese classification [26]. More precisely this class consists of the systems

$$
\mid \begin{aligned}
& \dot{x}=-y+\delta x+l x^{2}+m x y+n y^{2} \\
& \dot{y}=x+a x^{2}+b x y, \quad a \neq 0 .
\end{aligned}
$$

When $\delta=l=m=b=0, n \neq 0$ the system (0.4) is Hamiltonian and centrosymmetric with respect to the point $\left(-\frac{1}{2 a}, \frac{1}{2 n}\right)$. Then a simple corollary from Theorem 1 is the following one:

Corollary 1. - For $|a| \neq|n| \neq 0$ and small $\delta, l, m, b$, system (0.4) has no more than two limit cycles.

The techniques we use to get the results of the present paper is a combination of the techniques from [16] with some ideas from [11]. In particular we extensively use the notion and the properties of the centroid curve. (A centroid curve is formed by the mass centres of a continuous family of ovals within the level curves of $H$.) There are two centroid curves in the considered case, corresponding to two periodic annuli. In our study the elementary fact applies that each intersection point of the zero divergence line in $(0.1)$ with a centroid curve corresponds to a zero of $I(h)$ and hence to a limit cycle. Using the mutual position of the two centroid curves, Theorem 1 is easily seen to be a consequence of the fact that in the generic case each of the centroid curves has a non-zero curvature. A convexity argument is quite natural in situations where the expected number of cycles is two. Incidentally, a convexity idea has been used by Il'yashenko [18] in his paper about Abelian integrals arising in symmetric perturbations of Hamiltonian systems with symmetry of order two.

A more detailed sketch of our construction which we believe will help the reader to overcome easier the rather technical proof is given in the next section. 


\section{PRELIMINARIES AND OUTLINE OF THE PROOF}

We start with the definition of the centroid curve for any cubic Hamiltonian $H$ having a centre. It is easy to see that the integral $I(h)$ from $(0.2)$ can be written in the form

$$
I(h)=\iint_{\text {Int }(h)}(\alpha x+\beta y+\gamma) d x d y,
$$

where $\operatorname{Int}(h)$ is the region inside the oval $\delta(h)$ and $\alpha x+\beta y+\gamma=-f_{x}-g_{y}$. Define the integrals

$$
\begin{gathered}
X(h)=\iint_{\text {Int }(h)} x d x d y \\
Y(h)=\iint_{\text {Int }(h)} y d x d y, \quad M(h)=\iint_{I n t(h)} d x d y .
\end{gathered}
$$

Then the centroid point [10] of region $\operatorname{Int}(h)$ has coordinates $(\xi(h), \eta(h))$ where $\xi=X / M, \eta=Y / M$.

Definition 1.1. - Let $\left[h_{1}, h_{2}\right]$ be the maximal interval of existence of the oval $\delta(h)$ (including the separatrix cycle and the centre inside). Then the curve

$$
L=\left\{(\xi(h), \eta(h)): h \in\left[h_{1}, h_{2}\right]\right\}
$$

is called [16] the centroid curve of the family of ovals.

The importance of the concept of the centroid curve lies in the fact that its geometry contains the complete information about the possible zeros of each integral $I(h)=\alpha X(h)+\beta Y(h)+\gamma M(h)$ (i.e. for all values $\alpha, \beta, \gamma$ and all perturbations $f, g$ ), although the definition of $L$ depends only on $H$. We mention also the following obvious facts: a) $L$ is affine invariant; b) the endpoints of $L$ are the centroid $Z$ of the area bounded by the separatrix cycle and the centre $C$ lying inside; c) for non-generic Hamiltonians, $L$ is a line segment; d) $M(h)$ gives the area of $\operatorname{Int} \delta(h)$ and $T=M^{\prime}$ is the period function. It has been recently proved [8] that $T$ is monotone and hence $M^{\prime \prime} \neq 0$.

Specifying Definition 1.1 to the case of symmetric Hamiltonians $(0.3)$ we see that there are two continuous families of ovals $\delta(h)$ defined respectively in the intervals $\left[-h_{c},-h_{s}\right]$ and $\left[h_{s}, h_{c}\right]$, where $0<h_{s}<h_{c}$ are given by the formulas

$$
h_{c}=\frac{1}{3}\left[(1+\mu)^{3 / 2}+(1-\mu)^{3 / 2}\right], \quad h_{s}=\frac{1}{3}\left[(1+\mu)^{3 / 2}-(1-\mu)^{3 / 2}\right] .
$$


We shall denote by $L_{1}$ and by $L_{2}$ the corresponding centroid curves. We point out that in view of the symmetry of $H$ the curve $L_{2}$ is just $L_{1}$ rotated on an angle $\pi$. In the sequel we use $L$ to denote any of $L_{1}, L_{2}$. Further denote by $C_{1}, C_{2}$ the centres and by $S_{1}, S_{2}$ the saddles of the Hamiltonian (0.3). For later use we compute their coordinates, obtaining

$$
C_{1}=\left(x_{c}, y_{c}\right)=\left(\left(\frac{1+\sqrt{1-\mu^{2}}}{2}\right)^{1 / 2},-\left(\frac{1-\sqrt{1-\mu^{2}}}{2}\right)^{1 / 2}\right)
$$

and respectively $S_{1}=\left(x_{s}, y_{s}\right)=\left(-y_{c},-x_{c}\right), C_{2}=-C_{1}, S_{2}=-S_{1}$.

The following theorem sums up several properties of the centroid curve of generic Hamiltonians obtained in [16] and which we need in the present paper.

THEOREM 1.1. - Suppose that $H$ is generic. Then

(i) Near its endpoints the curve $L$ is regular, i.e. $\xi^{\prime 2}(h)+\eta^{\prime 2}(h) \neq 0$.

(ii) Near the endpoints of $L$ the curvature $\kappa$ is not zero and has the same sign.

It is not hard to see [16] that for a given perturbation $f, g$ in $(0.1)$ with $|\alpha|+|\beta| \neq 0$, the number of limit cycles in (0.1) for small $\varepsilon$ is equal to the number of the intersection points (counting the multiplicities) of $L$ with the line $\ell: \alpha x+\beta y+\gamma=0$, provided $L$ is regular. Moreover as $\varepsilon \rightarrow 0$ these limit cycles tend to the ovals $\delta(h)$ whose centroid points lie on $\ell$. Hence our goal would be achieved if we show that $L_{1}, L_{2}$ are regular and that any line intersects their union $\mathcal{L}=L_{1} \cup L_{2}$ in at most two points. In section 5 we prove the regularity of the centroid curves in the case of an arbitrary generic Hamiltonian with two centres, using Picard-Lefschetz theory [3]. The proof is similar to that in [16] and exploits ideas from [12] and [22]. Note that the regularity of $L$ as well as the nonvanishing of the curvature are affine invariant conditions. Most of our efforts will be concentrated to show that an arbitrary line $\ell$ intersects $L_{1}$ in no more than two points. Therefore the following theorem is the bulk of our construction:

THEOREM 1.2. - The curvature of the centroid curve $L_{1}$ at each point is non-zero.

Our plan to show the nonvanishing of the curvature is to start with a Hamiltonian $H$ for which we already know that. Such one can be obtained for example from the standard elliptic Hamiltonian $H_{0}=y^{2}+x^{3}-x$ via small perturbation in the direction of the symmetric Hamiltonians. This is done in section 6. From Theorem 1.1 we know that in the generic case the curvature near the endpoints of the centroid curve is always non-zero. 
This implies that varying the parameters in $H$ if the curvature acquires a zero this would be at least a double zero. It will correspond to a zero of multiplicity at least four of $I(h)$ provided $f, g$ are properly chosen ([16], Corollary 2.1). A simple but important fact is that the second derivative of $I$ and the first derivative of the period function $T$ satisfy Picard-Fuchs system of order two, and hence the ratio $w=I^{\prime \prime} / T^{\prime}$ satisfies a Riccati equation. This observation, used for the first time in [11], is based on the fact that the residua of the form $\omega=-f d y+g d x$ are linear in $h$ and therefore the second covariant derivative of the Gauss-Manin connection of $\omega$ has no residua. In section 2 of the paper we use these ideas to derive Riccati equation for $w$. Most of the present paper is devoted to a precise qualitative study of the Riccati equation satisfied by $w$. In particular we explore the properties of the zero isocline of the equation. On its hand to study the zero isocline we include it in a family of curves forming the level curves of a suitable Hamiltonian. We repeat the same procedure this time considering the horizontal and also the vertical isocline of the Hamiltonian system to obtain its phase portrait. From the properties of the zero isocline of the Riccati equation we find the possible behaviour of $w$. The upshot is that $w=I^{\prime \prime} / T^{\prime}$ (and hence $I^{\prime \prime}$ ) has no double zeros. All this analysis is contained in section 4 .

We have to emphasize that in section 4 we do not study $w$ for all values of the parameters $\alpha, \beta, \gamma$. We are interested only in values $\alpha, \beta, \gamma$ corresponding to lines $\ell$ tangent to $L_{1}$. In this case $w$ is subject to quite restrictive inequalities, which we obtain in section 3 . The proof of Theorem 1 is completed in section 7 by examining the mutual position of the two centroid curves.

In theory the same plan could be applied to all another cases (including the one studied in [16]). Unfortunately the computations in general become much more complicated. In spite of that we believe that the plan can be performed, eventually with some modifications. In this respect the present paper, apart of its own importance, can serve as a model for the general case.

\section{PICARD-FUCHS SYSTEM AND RICCATI EQUATION}

In this section we derive several differential equations satisfied by Abelian integrals. Our final goal will be the Riccati equation satisfied by $I^{\prime \prime} / M^{\prime \prime}$. For this we need to derive Picard-Fuchs system for the integrals $I_{0}, I_{-1}, I_{-2}$, defined below. 
Given $h \in\left(-h_{c},-h_{s}\right)$, we consider the level curve $H=x y^{2}+\mu y+$ $\frac{1}{3} x^{3}-x=h$, whose equation can be written also in the form

$$
\left(x y+\frac{\mu}{2}\right)^{2}=\frac{\mu^{2}}{4}+h x+x^{2}-\frac{x^{4}}{3} \equiv \mathcal{R}(x, h) \text {. }
$$

Put $z=x y+\mu / 2$ and define the integrals

$$
I_{k}(h)=\int_{\delta(h)} x^{k} z d x
$$

We have

$$
X(h)=\iint_{\text {Int } \delta(h)} x d x d y=-\int_{\delta(h)} z d x=-I_{0}
$$

and similarly $Y(h)=\frac{1}{2} \mu I_{-2}, M(h)=-I_{-1}$.

LEMMA 2.1. - The integrals $I_{0}, I_{-1}, I_{-2}$ satisfy the following system of equations

$$
\left\{\begin{array}{l}
\frac{4}{3} \mu^{2} I_{0}^{\prime \prime}+2 h I_{-1}^{\prime \prime}+\left(3 h^{2}-2 \mu^{2}\right) I_{-2}^{\prime \prime}-2 I_{-1}^{\prime}-h I_{-2}^{\prime}+I_{-2}=0 \\
2 h I_{0}^{\prime}+2\left(1+\frac{1}{3} \mu^{2}\right) I_{-1}^{\prime}+h I_{-2}^{\prime}-2 I_{0}-I_{-2}=0 \\
2 I_{0}^{\prime}+3 h I_{-1}^{\prime}+\mu^{2} I_{-2}^{\prime}-2 I_{-1}=0 .
\end{array}\right.
$$

Proof. - Express the left and the right hand-side of the identity

$$
\int_{\delta(h)} x^{k} \mathcal{R}^{\prime} z d x=-\frac{2 k}{3} \int_{\delta(h)} x^{k-1} \mathcal{R} z d x
$$

in terms of the functions $I_{k}, k=0, \pm 1, \ldots$ This gives a connection between these functions:

$$
\frac{k+6}{3} I_{k+3}=(k+3) I_{k+1}+\left(k+\frac{3}{2}\right) h I_{k}+\frac{k \mu^{2}}{4} I_{k-1} .
$$

Similarly using

$$
I_{k}^{\prime}=\int_{\delta(h)} \frac{x^{k+1}}{2 z} d x
$$


we obtain

$$
\begin{gathered}
I_{k}=\frac{\mu^{2}}{2} I_{k-1}^{\prime}+2 h I_{k}^{\prime}+2 I_{k+1}^{\prime}-\frac{2}{3} I_{k+3}^{\prime} \\
k I_{k-1}=-h I_{k-1}^{\prime}-2 I_{k}^{\prime}+\frac{4}{3} I_{k+2}^{\prime} .
\end{gathered}
$$

Using (2.3) with $k=-1$ and (2.4) with $k=0$ we get the third equation of (2.1). In the same manner applying (2.2) and (2.3) with $k=0$ and (2.4) with $k=-1$ we obtain the second equation of (2.1). At the end, if we eliminate $I_{1}$ and $I_{-3}$ from (2.2), (2.3), (2.4) with $k=-2$, we obtain the first equation.

Corollary 2.2. - The integrals $X, Y, M$ satisfy the system

$$
\left\{\begin{array}{l}
\frac{2}{3} \mu^{3} X^{\prime \prime}+\mu h M^{\prime \prime}-\left(3 h^{2}-2 \mu^{2}\right) Y^{\prime \prime}-\mu M^{\prime}+h Y^{\prime}-Y=0 \\
\mu h X^{\prime}+\mu\left(1+\frac{1}{3} \mu^{2}\right) M^{\prime}-h Y^{\prime}-\mu X+Y=0 \\
X^{\prime}+\frac{3}{2} h M^{\prime}-\mu Y^{\prime}-M=0 .
\end{array}\right.
$$

Now we are in a position to derive the Riccati equation satisfied by $w(h)=I^{\prime \prime}(h) / M^{\prime \prime}(h)$. Put $v=w-\gamma$. Then we can consider that $v=I^{\prime \prime} / M^{\prime \prime}$ where in $I(h)$ we have $\gamma=0$. Differentiate once the first two equations in (2.5) and twice the third one to get

$$
\left\{\begin{array}{l}
\frac{2}{3} \mu^{3} X^{\prime \prime \prime}+\mu h M^{\prime \prime \prime}-\left(3 h^{2}-2 \mu^{2}\right) Y^{\prime \prime \prime}-5 h Y^{\prime \prime}=0 \\
\mu h X^{\prime \prime}+\mu\left(1+\frac{1}{3} \mu^{2}\right) M^{\prime \prime}-h Y^{\prime \prime}=0 \\
X^{\prime \prime \prime}+\frac{3}{2} h M^{\prime \prime \prime}-\mu Y^{\prime \prime \prime}+2 M^{\prime \prime}=0
\end{array}\right.
$$

From these equations and from $I=\alpha X+\beta Y$ eliminate first $Y$ and then $X$. After solving with respect to $I^{\prime \prime \prime}$ and $M^{\prime \prime \prime}$ we obtain

$$
\left\{\begin{array}{l}
-3 h \Delta I^{\prime \prime \prime}=\left(5 h^{4}+B_{1} h^{2}\right) I^{\prime \prime}+\left(D h^{3}-E h\right) M^{\prime \prime} \\
-3 h \Delta M^{\prime \prime \prime}=A h^{3} I^{\prime \prime}+\left(4 h^{4}-B_{2} h^{2}-C\right) M^{\prime \prime} .
\end{array}\right.
$$


Here for shortness we have denoted

$$
\begin{aligned}
& \Delta=h^{4}-\frac{4}{9}\left(1+3 \mu^{2}\right) h^{2}+\frac{4}{9} \mu^{2}\left(1+\frac{1}{3} \mu^{2}\right)^{2}=\left(h^{2}-h_{c}^{2}\right)\left(h^{2}-h_{s}^{2}\right), \\
& A=-\frac{10\left(1-\mu^{2}\right)}{3 a}, \quad B_{1}=-\frac{10}{3} \mu\left(1+\frac{1}{3} \mu^{2}\right) \frac{b}{a}, \\
& B_{2}=\frac{4}{3}\left(1+3 \mu^{2}\right)-\frac{10}{3} \mu\left(1+\frac{1}{3} \mu^{2}\right) \frac{b}{a}, \\
& C=\frac{4}{3} \mu^{2}\left(1+\frac{1}{3} \mu^{2}\right)^{2}, \quad D=\frac{a}{1-\mu^{2}}\left[2 \mu\left(1+\frac{1}{3} \mu^{2}\right) \frac{b}{a}-\frac{2}{3}\left(1+3 \mu^{2}\right)\right], \\
& E=-\frac{2 a \mu\left(1+\frac{1}{3} \mu^{2}\right)}{3\left(1-\mu^{2}\right)}\left[5 \mu\left(1+\frac{1}{3} \mu^{2}\right) \frac{b^{2}}{a^{2}}-4\left(1+3 \mu^{2}\right) \frac{b}{a}+7 \mu\left(1+\frac{1}{3} \mu^{2}\right)\right], \\
& \text { where } \quad a=\alpha+\beta \mu, b=\beta+\alpha \mu .
\end{aligned}
$$

From (2.6) we derive the Riccati equation

$$
3 h \Delta v^{\prime}=A h^{3} v^{2}-\left(h^{4}+B h^{2}+C\right) v-D h^{3}+E h .
$$

Here $B=B_{1}+B_{2}$. Returning to the variable $w=v+\gamma$ and writing (2.8) as a system we finally get

Lemma 2.3. - The following Picard-Fuchs system is satisfied:

$$
\left\{\begin{array}{l}
\dot{w}=A h^{3}(w-\gamma)^{2}-\left(h^{4}+B h^{2}+C\right)(w-\gamma)-D h^{3}+E h \\
\dot{h}=3 h\left(h^{2}-h_{c}^{2}\right)\left(h^{2}-h_{s}^{2}\right) .
\end{array}\right.
$$

This is our basic system which we will study in the next two sections. In particular of a great importance for our analysis will be the properties of the zero isocline $\Gamma_{0}$ of (2.9), given by the points $(h, w)$ on the algebraic curve

$$
P(h, w) \equiv A h^{3}(w-\gamma)^{2}-\left(h^{4}+B h^{2}+C\right)(w-\gamma)-D h^{3}+E h=0 .
$$

We will often consider $\Gamma_{0}$ as the graph of the two-valued function $w_{0}^{ \pm}(h)$ determined by $P\left(h, w_{0}^{ \pm}(h)\right) \equiv 0$ ("+" is always assigned to the upper branch).

For later use denote by $\mathcal{D}_{0}$ the discriminant of the quadratic equation (2.10):

$$
\mathcal{D}_{0}=\left(h^{4}+B h^{2}+C\right)^{2}+4 A D h^{6}-4 A E h^{4} .
$$




\section{BASIC INEQUALITIES}

In this section we derive several inequalities concerning the parameters in (2.9) and the values of $w_{0}^{ \pm}$at the critical values of $H$, provided the line $\ell: \alpha x+\beta y+\gamma=0$ and the centroid curve $L_{1}$ satisfy some conditions listed below. These inequalities are crucial in our analysis of (2.9).

Through this and the next section we suppose the following:

(U) (i) $\ell$ is a tangent to the centroid curve $L_{1}$ at an internal point $(\xi(h), \eta(h))$;

(ii) the curvature of $L_{1}$ does not change the sign (but may vanish);
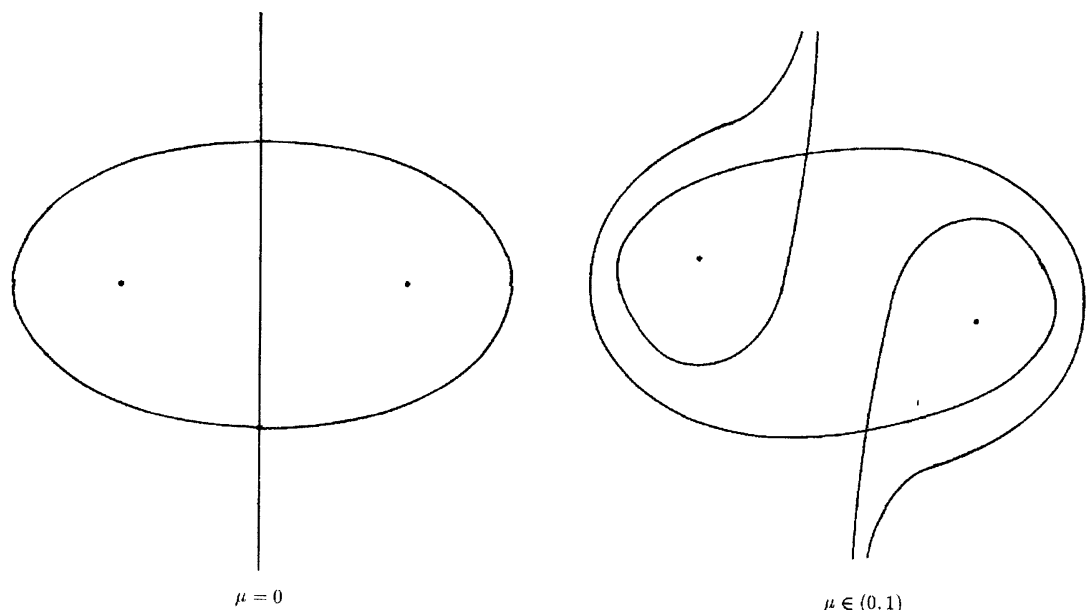

$\mu \in(0,1)$

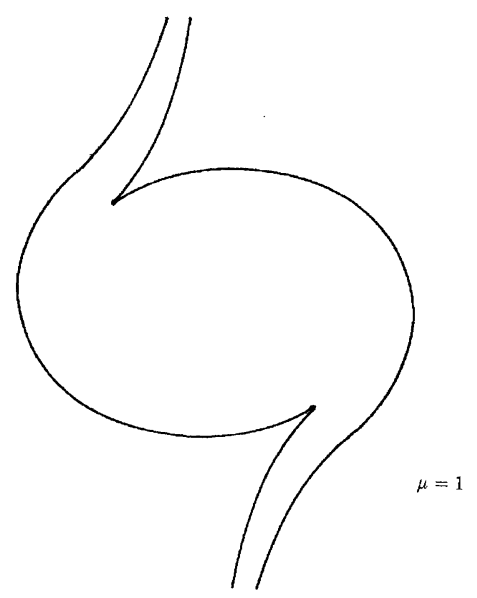

Fig. 1. 
(iii) running $L_{1}, \ell$ rotates on an angle less than $\pi$.

We start with certain restrictions for the coefficients of the line $\ell$ imposed by (U).

LEMma 3.1. - The coefficients $\alpha, \beta, \gamma$ (multiplied if needed by -1) satisfy the inequalities

$$
\gamma>0, \quad \alpha+\beta \mu<0, \quad \beta+\alpha \mu>0 .
$$

Proof. - We are going to prove that the angular coefficient of $\ell$ satisfies the inequalities

$$
\mu<-\frac{\alpha}{\beta}<\frac{1}{\mu}
$$

If this was done and assuming that $\beta>0$ we immediately get the last two inequalities in (3.1). Moreover the loop through $S_{1}$ is placed in the half-plane $x>0$ and it is easily verified that if $\left(x, y_{1}\right)$ and $\left(x, y_{2}\right)$ are points on $\delta(h), h \in\left(-h_{c},-h_{s}\right)$, then $y_{1}+y_{2}<0$. Therefore $L_{1}$ is situated in the fourth quadrant and since $\alpha<0<\beta$ the equality $\alpha \xi(h)+\beta \eta(h)+\gamma=0$ is possible only if $\gamma>0$.

In order to prove (3.2) we need another normal form

$$
H=\frac{x^{2}+y^{2}}{2}-\frac{x^{3}}{3}+\frac{\lambda y^{3}}{3}, \quad \lambda \in(0,1)
$$

of the Hamiltonian. In these coordinates $L_{1}$ corresponds to values $h \in\left[0, \frac{1}{6}\right]$. Having in mind $(\mathrm{U})$, the proof consists of finding the equations of the tangents at the endpoints of $L_{1}$. According to Corollary 3.4 from [16] the equation of the tangent $\ell_{c}$ of $L_{1}$ at the centre $C_{1}(0,0)$ is $y=-\lambda x$ (see Fig. 2). The tangent $\ell_{s}$ at the other endpoint goes through the saddle $S_{1}=(1,0)$ and through the centroid of the loop area $Z_{1}$ [16], but the coordinates of $Z_{1}$ are given by extremely long formulas. Because of this we use another line instead of $\ell_{s}$. The saddle-loop is determined by the equation $H=\frac{1}{6}$. The line $x=\frac{1}{2}$ divides the region inside the saddleloop into two parts $\Lambda_{c}$ and $\Lambda_{s}$ containing respectively the centre and the saddle. Obviously the reflection of $\Lambda_{s}$ through the line $x=\frac{1}{2}$ is contained in $\Lambda_{c}$. This means that $\xi\left(\frac{1}{6}\right)<\frac{1}{2}$. Then using (U) and results from [16] yields that $Z_{1}=\left(\xi\left(\frac{1}{6}\right), \eta\left(\frac{1}{6}\right)\right)$ is located inside the triangle with vertices $(0,0),\left(\frac{1}{2}, 0\right),\left(\frac{1}{2},-\frac{\lambda}{2}\right)$. Let $m$ be the line symmetric to $\ell_{c}$ with respect to the line $x=\frac{1}{2}$, i.e. $m$ has an equation $y=\lambda(x-1)$. Then the angular coefficient $k$ of an arbitrary tangent $\ell$ at an interior point of $L_{1}$ satisfies 


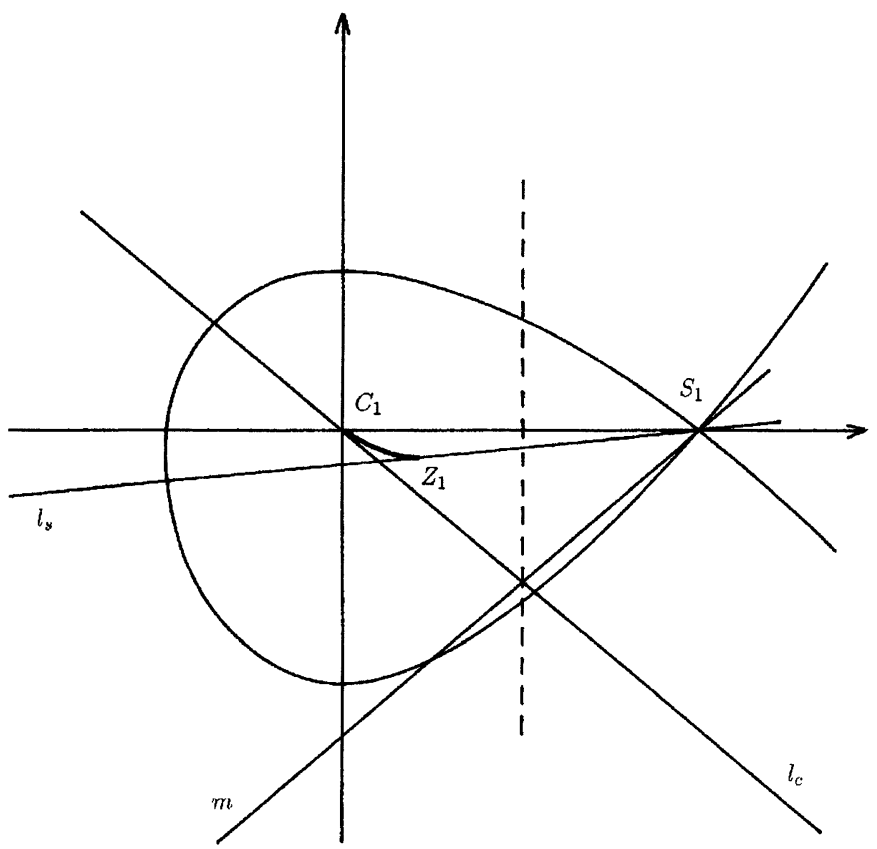

Fig. 2.

the inequalities $-\lambda<k<\lambda$. In order to return to the coordinate system in which the normal form is $(0.3)$ we change the variables $(x, y) \rightarrow\left(x_{1}, y_{1}\right)$,

$$
x=\frac{1}{2}\left(-\frac{x_{1}+y_{1}}{\sqrt{1-\mu}}+1\right), \quad y=\frac{1}{2}\left(\frac{1+\mu}{1-\mu}\right)^{\frac{3}{4}}\left(\frac{x_{1}-y_{1}}{\sqrt{1+\mu}}-1\right),
$$

where

$$
\mu=\frac{1-\lambda^{\frac{4}{3}}}{1+\lambda^{\frac{4}{3}}}
$$

In this coordinate system the equations of $\ell_{c}$ and $m$ become respectively (we omit the subscript 1)

$$
\left\{\begin{array}{l}
\ell_{c}: \quad y=\mu x-\frac{(1+\mu)^{\frac{3}{2}}-(1-\mu)^{\frac{3}{2}}}{2} \\
m: \quad y=\frac{x}{\mu}-\frac{(1+\mu)^{\frac{3}{2}}-(1-\mu)^{\frac{3}{2}}}{2 \mu} .
\end{array}\right.
$$

Hence the angular coefficient $k=-\alpha / \beta$ of any tangent line satisfies $\mu<k<1 / \mu$. 
As a direct consequence of (2.7) and (3.1) we obtain

Corollary 3.2. - The constants $A, B, C, D, E$ in (2.9) are positive.

LEMMA 3.3. - (i) The values of $w_{0}^{ \pm}(h)$ at the ends of the interval $\left[-h_{c},-h_{s}\right]$ have the following signs:

$$
\begin{aligned}
& w_{0}^{+}\left(-h_{s}\right)=\alpha x_{s}+\beta y_{s}+\gamma<0, \\
& w_{0}^{+}\left(-h_{c}\right)>0, \\
& w_{0}^{-}\left(-h_{c}\right)=\alpha x_{c}+\beta y_{c}+\gamma<0 .
\end{aligned}
$$

(ii) The derivatives satisfy

$$
\frac{d}{d h} w_{0}^{ \pm}\left(-h_{s}\right)<0, \quad \frac{d}{d h} w_{0}^{ \pm}\left(-h_{c}\right)<0 .
$$

Proof. - (i) Using the explicit formulas for $\Delta, A, B$ etc. we express the discriminant $\mathcal{D}_{0}$ from $(2.11)$ in the form

$$
\begin{gathered}
\mathcal{D}_{0}=\Delta\left[h^{4}+\left(12\left(1+3 \mu^{2}\right)-40 \mu\left(1+\frac{1}{3} \mu^{2}\right) \frac{b}{a}\right) h^{2}\right. \\
\left.+4 \mu^{2}\left(1+\frac{1}{3} \mu^{2}\right)^{2}\right]+\frac{64}{9}\left(1-\mu^{2}\right)^{3} h^{4}
\end{gathered}
$$

Also we have

$$
h^{4}+B h^{2}+C=3 \Delta-2 h^{4}+\left[\frac{8}{3}\left(1+3 \mu^{2}\right)-\frac{20}{3} \mu\left(1+\frac{1}{3} \mu^{2}\right) \frac{b}{a}\right] h^{2} .
$$

This gives after obvious calculations

$$
\begin{aligned}
& \left.w_{0}^{ \pm}\right|_{\Delta=0}=\gamma+\frac{1}{2 A h^{3}}\left[h^{4}+B h^{2}+C \mp \sqrt{\mathcal{D}_{0}}\right]_{\mid \Delta=0}=\gamma+\frac{1}{2 A h^{3}} \\
& \quad \times\left\{-2 h^{4}+\left[\frac{8}{3}\left(1+3 \mu^{2}\right) \mp \frac{8}{3}\left(1-\mu^{2}\right)^{\frac{3}{2}}\right.\right. \\
& \left.\left.\quad-\frac{20}{3} \mu\left(1+\frac{1}{3} \mu^{2}\right) \frac{b}{a}\right] h^{2}\right\}=\gamma \\
& \quad+\frac{1}{10\left(1-\mu^{2}\right) h}\left\{a\left[3 h^{2}-4-12 \mu^{2} \pm 4\left(1-\mu^{2}\right)^{\frac{3}{2}}\right]+10 b \mu\left(1+\frac{1}{3} \mu^{2}\right)\right\} .
\end{aligned}
$$


Further, with the help of (2.7) and the formulas for $x_{c}, y_{c}, h_{c}$ from section 1 we obtain via straightforward computations that $w_{0}^{-}\left(-h_{c}\right)=\alpha x_{c}+\beta y_{c}+\gamma$. In the same way we compute $w_{0}^{+}\left(-h_{s}\right)$. At the end

$$
\begin{aligned}
& w_{0}^{+}\left(-h_{c}\right)=w_{0}^{-}\left(-h_{c}\right)+\frac{8\left(1-\mu^{2}\right)^{\frac{3}{2}}}{3 A h_{c}} \\
& =\alpha x_{c}+\beta y_{c}+\gamma-\frac{4 a\left(1-\mu^{2}\right)^{\frac{1}{2}}}{5 h_{c}}=\alpha \bar{x}_{c}+\beta \bar{y}_{c}+\gamma,
\end{aligned}
$$

where

$$
\bar{x}_{c}=x_{c}-\frac{4\left(1-\mu^{2}\right)^{\frac{1}{2}}}{5 h_{c}}, \quad \bar{y}_{c}=y_{c}-\frac{4 \mu\left(1-\mu^{2}\right)^{\frac{1}{2}}}{5 h_{c}} .
$$

From (3.4) it is clear that the point $\left(\bar{x}_{c}, \bar{y}_{c}\right)$ is lying on the tangent $\ell_{c}$. We will show further that $\bar{x}_{c}<x_{s}$. Really $x_{s}=-y_{c}$ and $\bar{x}_{c}+y_{c}=$ $x_{c}+y_{c}-\frac{4}{5 h_{c}} \sqrt{1-\mu^{2}}$. But $x_{c}+y_{c}<\frac{4}{5 h_{c}} \sqrt{1-\mu^{2}}$ is equivalent to

$$
\left(x_{c}+y_{c}\right)^{2}=1-\mu<\frac{16\left(1-\mu^{2}\right)}{25 h_{c}^{2}} \Leftrightarrow h_{c}^{2}<\frac{16(1+\mu)}{25} .
$$

The last inequality easily follows from $h_{c}^{2}<\frac{2}{9}(2+3 \mu)<\frac{16}{25}(1+\mu)$.

The inequality $\bar{x}_{c}<x_{s}$ shows that $\left(\bar{x}_{c}, \bar{y}_{c}\right)$ is above any tangent $\ell$ to $L_{1}$ which gives that $w_{0}^{+}\left(-h_{c}\right)=\alpha \bar{x}_{c}+\beta \bar{y}_{c}+\gamma>0$. The other two inequalities in (i) follow from the fact that both the centre $\left(x_{c}, y_{c}\right)$ and the saddle $\left(x_{s}, y_{s}\right)$ are below any tangent $\ell$.

(ii) Evaluating the derivatives of $w_{0}^{ \pm}$at $\Delta=0$ we get

$$
\begin{aligned}
\left.\frac{d w_{0}^{ \pm}}{d h}\right|_{\Delta=0}= & \frac{1}{2 A h^{3}}\left(3 \Delta^{\prime}-8 h^{3}+2\left[\frac{8}{3}\left(1+3 \mu^{2}\right)\right.\right. \\
& \left.\left.-\frac{20}{3} \mu\left(1+\frac{1}{3} \mu^{2}\right) \frac{b}{a}\right] h \mp \frac{\mathcal{D}_{0}^{\prime}}{2 \sqrt{\mathcal{D}_{0}}}\right)-\left.\frac{3}{h}\left(w_{0}^{ \pm}-\gamma\right)\right|_{\Delta=0} \\
= & \frac{1}{2 A h^{3}}\left\{3 \Delta^{\prime} \mp \frac{\mathcal{D}_{0}^{\prime}}{2 \sqrt{\mathcal{D}_{0}}}+h\left[-2 h^{2}-\frac{8}{3}\left(1+3 \mu^{2}\right) \pm 8\left(1-\mu^{2}\right)^{\frac{3}{2}}\right.\right. \\
& \left.\left.+\frac{20}{3} \mu\left(1+\frac{1}{3} \mu^{2}\right) \frac{b}{a}\right]\right\}\left.\right|_{\Delta=0} .
\end{aligned}
$$

Write $\mathcal{D}_{0}$ in the form

$$
\begin{aligned}
\mathcal{D}_{0}=9 \Delta^{2} & +8 \Delta\left[-h^{2}+2\left(1+3 \mu^{2}\right)-5 \mu\left(1+\frac{1}{3} \mu^{2}\right) \frac{b}{a}\right] h^{2} \\
& +\frac{64}{9}\left(1-\mu^{2}\right)^{3} h^{4} .
\end{aligned}
$$


This gives

$$
\left.\frac{\mathcal{D}_{0}^{\prime}}{2 \sqrt{\mathcal{D}_{0}}}\right|_{\Delta=0}=\frac{\Delta^{\prime}\left[-h^{2}+2\left(1+3 \mu^{2}\right)-5 \mu\left(1+\frac{1}{3} \mu^{2}\right) \frac{b}{a}\right]+\frac{32}{9}\left(1-\mu^{2}\right)^{3} h}{\frac{2}{3}\left(1-\mu^{2}\right)^{\frac{3}{2}}} .
$$

Calculation the values of $\Delta^{\prime}$ at $-h_{s}$ and $-h_{c}$ yields:

$$
\Delta^{\prime}\left(-h_{s}\right)=\frac{8}{9} h_{s}\left(1-\mu^{2}\right)^{\frac{3}{2}}, \Delta^{\prime}\left(-h_{c}\right)=-\frac{8}{9} h_{c}\left(1-\mu^{2}\right)^{\frac{3}{2}} .
$$

Finally we substitute $h=-h_{c}$ in the above formulas and via direct computations find

$$
\begin{gathered}
\frac{d w_{0}^{-}}{d h}\left(-h_{c}\right)=\frac{a}{2\left(1-\mu^{2}\right)}<0, \\
\frac{d w_{0}^{+}}{d h}\left(-h_{c}\right)=-\frac{36 h_{s}^{2}+h_{c}^{2}-20 \mu\left(1+\frac{1}{3} \mu^{2}\right) \frac{b}{a}}{3 A h_{c}^{2}}<0 .
\end{gathered}
$$

Repeating the above computation for $h=-h_{s}$ we obtain

$$
\begin{gathered}
\frac{d w_{0}^{+}}{d h}\left(-h_{s}\right)=\frac{a}{2\left(1-\mu^{2}\right)}<0, \frac{d w_{0}^{-}}{d h}\left(-h_{s}\right) \\
=-\frac{36 h_{c}^{2}+h_{s}^{2}-20 \mu\left(1+\frac{1}{3} \mu^{2}\right) \frac{b}{a}}{3 A h_{s}^{2}}<0
\end{gathered}
$$

thus proving the lemma.

\section{THE ZERO ISOCLINE OF THE RICCATI EQUATION}

In this section we list a number of properties of the zero isocline $\Gamma_{0}$ of (2.9) supposing (U) hold. In particular decisive for our purposes will be the number and the mutual positions of the minima and maxima of $w_{0}^{ \pm}(h)$. We start with the following lemma.

Lemma 4.1. - (i) The curve $\Gamma_{0}$ is centrosymmetric with respect to the point $(0, \gamma)$.

(ii) $\Gamma_{0}$ has no compact components.

Proof. - Assertion (i) is obvious. To prove (ii) we observe that the discriminant $\mathcal{D}_{0}$ given by $(2.11)$ can have no more than two zeros for $h<0$. Together with the behaviour near $h=0$ (see the next lemma), this eliminates the existence of compact components. 
Next we study certain asymptotic properties of $w_{0}^{ \pm}(h), h<0$.

Lemma 4.2. - (i) The function $w_{0}^{+}(h)$ has the following asymptotic expansions

$$
\begin{gathered}
w_{0}^{+}(h)=\gamma+\frac{E h}{C}+O\left(h^{3}\right) \text { when } h \rightarrow-0, \\
w_{0}^{+}(h)=\gamma-\frac{D}{h}+O\left(h^{-3}\right) \text { when } h \rightarrow-\infty .
\end{gathered}
$$

(ii) The function $w_{0}^{-}(h)$ has the following asymptotic expansions:

$$
\begin{gathered}
w_{0}^{-}(h)=\frac{C}{A h^{3}}+O\left(\frac{1}{h}\right) \quad \text { when } h \rightarrow-0, \\
w_{0}^{-}(h)=\frac{h}{A}+\gamma+O\left(\frac{1}{h}\right) \quad \text { when } h \rightarrow-\infty .
\end{gathered}
$$

Proof. - Direct computations.

Our further plan is to consider the entire family of level curves

$$
\Gamma_{p}=\{(h, w): P(h, w)=p\}
$$

which is tantamount to the phase portrait of the Hamiltonian system

$$
\begin{aligned}
\dot{h}= & P_{w}=2 A h^{3}(w-\gamma)-\left(h^{4}+B h^{2}+C\right) \\
\dot{w}= & -P_{h}=-\left[3 A h^{2}(w-\gamma)^{2}\right. \\
& \left.-\left(4 h^{3}+2 B h\right)(w-\gamma)-3 D h^{2}+E\right] .
\end{aligned}
$$

This is because the discriminant $\mathcal{D}_{0}$ is either positive or can change the sign depending on the parameter values in (2.11). Consequently $\Gamma_{0}$ can bifurcate and all possible situations should be occurred in the family $\left\{\Gamma_{p}: p \in \mathbb{R}\right\}$. Again for simplicity of the notation we can consider the portrait for $\gamma=0$, the general case being a mere translation $w \rightarrow w+\gamma$.

Lemma 4.3. - System (4.1) has exactly one singular point $S_{0}$ in the half-plane $h<0$. The point $S_{0}$ is a saddle.

Proof. - From the equations $P_{w}=P_{h}=0$ eliminate $w$ and put $h^{2}=z$ to obtain the equation

$$
5 z^{4}+6(B+2 A D) z^{3}+\left(B^{2}+2 C-4 A E\right) z^{2}-2 B C z-3 C^{2}=0 .
$$

Obviously this equation has at least one positive root $z_{0}$. The analysis of the coefficient alternations and Descartes' criterion rules out the existence 
of other positive roots. As the Poincaré index of the field in (4.1) cannot exceed 0 , using the symmetry yields that $S_{0}$ is a saddle point.

Denote by $h_{0}=-\sqrt{z_{0}}$ the abscissa of the saddle $S_{0}$. Our further analysis will concentrate mainly on study of two of the isoclines of (4.1), namely the horizontal one $\mathcal{H}=\left\{P_{h}=0\right\}$ and the vertical one $\mathcal{V}=\left\{P_{w}=0\right\}$. The properties of $\mathcal{V}$ we need are easy to obtain and are summed up in the following lemma:

LEMMA 4.4. - (i) The curve $\mathcal{V}$ is concave in the half-plane $h<0$.

(ii) Considered as a graph of $w_{V}(h)=\left(h^{4}+B h^{2}+C\right) / 2 A h^{3}$ its only maximum is at $h_{V}=-\sqrt{\frac{1}{2}\left(B+\sqrt{B^{2}+12 C}\right)}$.

(iii) The asymptotics hold

$$
\lim _{h \rightarrow-0} h^{3} w_{V}(h)=\frac{C}{2 A}, \quad \lim _{h \rightarrow-\infty} \frac{w_{V}(h)}{h}=\frac{1}{2 A} .
$$

Proof. - Direct computation.

The horizontal isocline $\mathcal{H}$ is more complicated for studying. Moreover it bifurcates as the parameters vary. To describe its properties we denote by $w_{H}^{+}(h), w_{H}^{-}(h)$ the two roots of the equation $P_{h}(h, w)=0, h<0$.

LEMMA 4.5. - (i) Let $B^{2}-3 A E \geq 0$. Then $\mathcal{H}$ consists of two separate curves given by the graphs of $w_{H}^{+}(h), w_{H}^{-}(h), h<0$. Their asymptotics are the following

$$
\left\{\begin{array}{l}
\lim _{h \rightarrow-0} h w_{H}^{ \pm}=\frac{B \mp \sqrt{B^{2}-3 A E}}{3 A}, \\
\lim _{h \rightarrow-\infty} h w_{H}^{+}=-\frac{3 D}{4}, \quad \lim _{h \rightarrow-\infty} \frac{w_{H}^{-}}{h}=\frac{4}{3 A} .
\end{array}\right.
$$

Each of the functions $w_{H}^{ \pm}(h)$ has exactly one maximum and no minima.

(ii) Let $B^{2}-3 A E<0$. Then the two graphs of $w_{H}^{ \pm}$meet at a finite point thus forming geometrically one smooth curve $\mathcal{H}$. For $h \rightarrow-\infty$ the two branches have the same asymptotics as in (4.2). The function $w_{H}^{+}(h)$ has always one maximum. The function $w_{H}^{-}(h)$ has either one minimum and one maximum or no extrema, the latter case including also the possibility of a collision of the maximum and minimum.

Proof. - The asymptotics are computed straightforward from the formulas

$$
\begin{gathered}
w_{H}^{ \pm}(h)=\frac{2 h^{2}+B \mp \sqrt{\mathcal{D}}}{3 A h}, \\
\mathcal{D}=4 h^{4}+(4 B+9 A D) h^{2}+\left(B^{2}-3 A E\right) .
\end{gathered}
$$


When $B^{2}-3 A E \geq 0$ both $w_{H}^{ \pm}(h)$ exist for all $h<0$. When $B^{2}-3 A E<0$ the discriminant $\mathcal{D}$ has a simple zero at a negative value $h=\bar{h}$, i.e. $w_{H}^{+}(\bar{h})=w_{H}^{-}(\bar{h})$ and both $w_{H}^{ \pm}(h)$ are defined for $h \in(-\infty, \bar{h}]$. At the end computing the derivative of $w_{H}^{ \pm}(h)$ we obtain:

$$
\frac{d w_{H}^{ \pm}}{d h}=\frac{\left(2 h^{2}-B\right) \sqrt{\mathcal{D}} \mp\left(4 h^{4}+3 A E-B^{2}\right)}{3 A h^{2} \sqrt{\mathcal{D}}} .
$$

Putting $h^{2}=z$ this yields a cubic equation for all extrema:

$$
12 D z^{3}-12(B D+E) z^{2}+B(3 B D+4 E) z+E\left(B^{2}-3 A E\right)=0 .
$$

In the case $B^{2}-3 A E \geq 0$ the asymptotics (4.2) say that each of $w_{H}^{ \pm}(h)$ has at least one maximum. As (4.3) has at most two positive solutions, this proves (i).

In the case $B^{2}-3 A E<0$ the equation (4.3) has either one or three positive solutions. Using the asymptotics (4.2) and the behaviour near $\bar{h}$ show that $w_{H}^{+}(h)$ has at least one maximum while $w_{H}^{-}(h)$ either has no extrema or has one maximum and one minimum. To obtain more precise information, we observe that the derivative of $w_{H}^{+}$is negative for $z<B / 2$ and the derivative of $w_{H}^{-}$is positive for $z>B / 2$. If $F(z)$ denotes the polynomial in (4.3), we have $F\left(\frac{B}{2}\right)=-3 A E^{2}<0, F^{\prime}\left(\frac{B}{2}\right)=-8 B E<0$. Hence (4.3) has exactly one zero greater than $B / 2$. This yields that $w_{H}^{+}$has always exactly one extremum (maximum). Lemma 4.5 is proved.

We will denote the two branches of $\mathcal{H}$ by $\mathcal{H}^{+}$and $\mathcal{H}^{-}$.

Our next objective is to locate the saddle $S_{0}$ with respect to the extrema of $w_{H}^{ \pm}$. Before that we need more refine study of $\mathcal{H}$, which we do again by considering the whole family of curves $P_{h}=$ const and their horizontal and vertical isoclines $\mathcal{H}_{H}=\left\{P_{h h}=0\right\}$ and $\mathcal{H}_{V}=\left\{P_{h w}=0\right\}$. Denote also by $\mathcal{H}_{H}^{ \pm}$the two branches of $\mathcal{H}_{H}$ (see Fig. 3). As above we consider these curves as graphs of the corresponding functions $w_{H}^{ \pm}(h)$ and $w_{H V}(h), h<0$.

Lemma 4.6. - (i) The curves $\mathcal{H}_{H}^{-}$and $\mathcal{H}_{V}$ are concave. Each of $\mathcal{H}_{H}^{ \pm}$has a maximum at $h_{H H}=-\sqrt{\frac{B}{6}}$ and $\mathcal{H}_{V}$ has a maximum at $h_{H V}=-\sqrt{\frac{B}{2}}$.

(ii) The curves $\mathcal{H}_{H}$ and $\mathcal{H}_{V}$ have no common point and $\mathcal{H}_{V}$ is situated between the two branches of $\mathcal{H}_{H}$.

Proof. - Direct computation.

Lemma 4.7. - (i) The curves $\mathcal{V}$ and $\mathcal{H}_{V}$ intersect at the unique point of maximum of $\mathcal{V}$. 


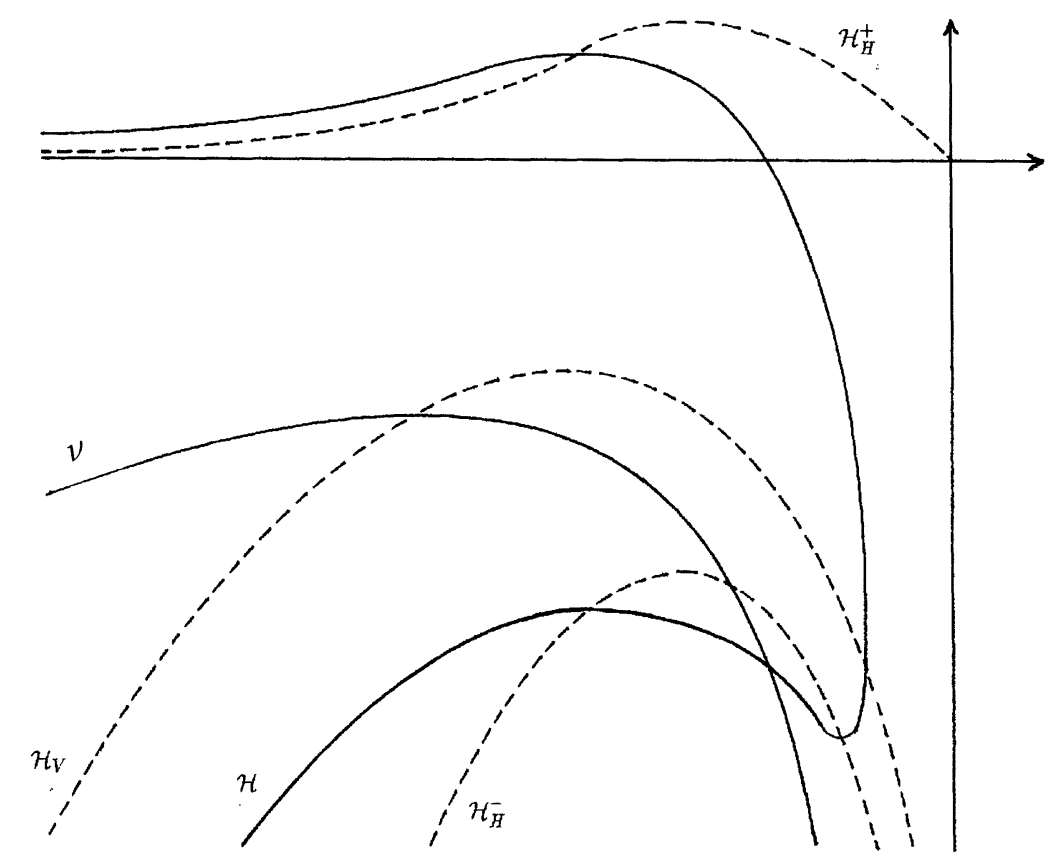

Fig. 3.

(ii) The curves $\mathcal{V}$ and $\mathcal{H}_{H}^{+}$have no common point, $\mathcal{V}$ and $\mathcal{H}_{H}^{-}$intersect at a unique point $S_{*}$ with an abscissa $h_{*}$ satisfying

$$
h_{H H}<h_{*}<0 \text {. }
$$

(iii) The saddle $S_{0}$ is on the lower branch of $\mathcal{H}$. Its abscissa $h_{0}$ satisfies

$$
h_{H V}<h_{0}<0 \text {. }
$$

Proof. - (i) is obvious. (ii) To prove the existence and uniqueness of $S_{*}$ we argue as above in establishing the existence of the saddle $S_{0}$. For the proof of the inequality $h_{H H}<h_{*}$ we first take the asymptotic values of the functions $w_{H}^{-}{ }_{H}(h)$ and $w_{V}(h)$ when $h \rightarrow-0$, namely

$$
w_{H H}^{-}(h) \sim \frac{B}{3 A h}, \quad w_{V}(h) \sim \frac{C}{2 A h^{3}} .
$$


This shows that $w_{H H}^{-}(h)>w_{V}(h)$ near $h=-0$. On the other hand we can see that $w_{H H}^{-}\left(h_{H H}\right)<w_{V}\left(h_{H H}\right)$ which in details reads

$$
-\frac{2 B+\sqrt{4 B^{2}+6 A B D}}{A \sqrt{6 B}}<-\frac{7 B^{2}+36 C}{2 A B \sqrt{6 B}}
$$

or $\left(3 B^{2}+36 C\right)^{2}<4 B^{2}\left(4 B^{2}+6 A B D\right)$. Using the expressions for $A, B, C, D$ we easily verify that $12 C<B^{2}, B<A D$. Then

$$
\left(3 B^{2}+36 C\right)^{2}<36 B^{4}<4 B^{2}\left(4 B^{2}+5 A B D\right)<4 B^{2}\left(4 B^{2}+6 A B D\right) .
$$

This proves (4.4).

(iii) We follow essentially the same argument, showing that

$$
w_{H}^{-}\left(h_{H V}\right)<w_{V}\left(h_{H V}\right) .
$$

This condition is equivalent to $\left(B^{2}+12 C\right)^{2}<8 B^{2}\left(8 B^{2}+9 A B D-6 A E\right)$ which follows from $12 C<B^{2}$ and the easily verified inequality $A E<B^{2}$. The inequality (4.6) we proved implies that $\mathcal{V}$ and the lower branch $\mathcal{H}^{-}$ intersect at a point $S_{0}$ with an abscissa $h_{0}$ satisfying (4.5). Really if $B^{2}-3 A E \geq 0$ then (4.2) and Lemma 4.4 (iii) give that $w_{H}^{-}(h)>w_{V}(h)$ near $h=-0$. If $B^{2}-3 A E<0$ then (4.6) implies that $h_{H V}<\bar{h}$, where $\bar{h}$ is the zero of $\mathcal{D}$ from Lemma 4.5 (ii). Hence $w_{H}^{-}(\bar{h})=w_{H V}(\bar{h})>w_{V}(\bar{h})$ because $\bar{h}>h_{H V}>h_{V}$ and $w_{H V}(h)>w_{V}(h)$ for $h>h_{V}$, the latter a consequence of (i), Lemma 4.4 (ii) and Lemma 4.6 (i). Thus $h_{H V}<h_{0}<\bar{h}$ and (iii) is proved.

Another property of $\mathcal{H}$ and $\mathcal{V}$ we need is the following

Lemma 4.8. - Suppose that the function $w_{H}^{-}(h)$ has a maximum at $h_{H}$. Then $h_{H}<h_{0}$ (The saddle $S_{0}$ is to the right of the maximum of $w_{H}^{-}(h)$ ).

Proof. - The extrema of $\mathcal{H}^{-}$lie on $\mathcal{H}_{H}^{-}$and $\mathcal{H}_{H}^{-}$itself is a concave curve with a maximum at a point with an abscissa $h_{H H}$. Since $w_{H H}^{-}(h)<$ $w_{H}^{-}(h) \rightarrow-\infty$ for $h \rightarrow-\infty$ this yields that the maximum of $\mathcal{H}^{-}$(if it exists) has an abscissa $h_{H}<h_{H H}$ and moreover $w_{H}^{-}(h)<w_{H}^{-}(h)$ for $h<h_{H}$. Suppose that $h_{0}<h_{H}$. Then $w_{H H}^{-}\left(h_{0}\right)<w_{H}^{-}\left(h_{0}\right)=w_{V}\left(h_{0}\right)$ and $w_{H}^{-}\left(h_{H}\right)=w_{H}^{-}\left(h_{H}\right)>w_{V}\left(h_{H}\right)$ because $w_{H}^{-}(h)>w_{V}(h)$ for $h>h_{0}$. Therefore the intersection point $S_{*}$ of $\mathcal{V}$ and $\mathcal{H}_{H}^{-}$has an abscissa $h_{*}$ with $h_{0}<h_{*}<h_{H}<h_{H H}-$ a contradiction with (4.4). This proves the lemma.

In order to make the facts proved in Lemmas 4.3-4.8 more applicable, below we list all needed points of $\mathcal{H}$ and $\mathcal{V}$ with their coordinates and characteristics: 
$S_{0}\left(h_{0}, w_{H}^{-}\left(h_{0}\right)\right)$ - the saddle point, $S_{0}=\mathcal{H} \cap \mathcal{V}$,

$S_{H}^{+}\left(h_{H}^{+}, w_{H}^{+}\left(h_{H}^{+}\right)\right)$- the point of maximum of $\mathcal{H}^{+}$,

$S_{V}\left(h_{V}, w_{V}\left(h_{V}\right)\right)$ - the point of maximum of $\mathcal{V}$,

$S_{H}\left(h_{H}, w_{H}^{-}\left(h_{H}\right)\right)$ - the point of maximum of $\mathcal{H}^{-}$,

$S_{H}^{-}\left(h_{H}^{-}, w_{H}^{-}\left(h_{H}^{-}\right)\right) \quad$ - the point of minimum of $\mathcal{H}^{-}$,

$\bar{S}\left(\bar{h}, w_{H}^{ \pm}(\bar{h})\right)$ - the point where $\mathcal{H}^{+}$meets $\mathcal{H}^{-}$.

(The last three points do not exist necessarily.)

Now we are able to describe in details the picture of the minima and maxima of $w_{0}^{ \pm}(h)$.

Lemma 4.9. - (i) Let $\mathcal{D}_{0} \geq 0$ for any $h$. Then the function $w_{0}^{+}(h), h<0$ has exactly one maximum and one minimum.

(ii) Let $\mathcal{D}_{0}<0$ for $h \in\left(h^{-}, h^{+}\right), h^{-}<h^{+}<0$. Then a) for $h \in\left(-\infty, h^{-}\right]$the function $w_{0}^{+}(h)$ has exactly one maximum and the function $w_{0}^{-}(h)$ has exactly one maximum and one minimum; $b$ ) for $h \in\left[h^{+}, 0\right)$ the function $w_{0}^{+}(h)$ has exactly one maximum and one minimum.

Proof. - The separatrices $\mathcal{H}$ and $\mathcal{V}$ divide the half plane $h<0$ into four open domains if $B^{2}-3 A E<0$ and into five open domains otherwise. Let us denote by $\mathcal{H}_{1}$ and $\mathcal{V}_{1}$ the parts of $\mathcal{H}^{-}$and $\mathcal{V}$ respectively which are to the left of the saddle $S_{0}$ and by $\mathcal{H}_{2}$ and $\mathcal{V}_{2}$ - the rest part of $\mathcal{H}$ and $\mathcal{V}$. Analytically (for example) we have $\mathcal{H}_{1}=\left\{\left(h, w_{H}^{-}(h)\right): \quad h<h_{0}\right\}$ and similarly for the other arcs. In the case where $B^{2}-3 A E \geq 0, \mathcal{H}_{2}$ itself consists of two separate curves, one of them is $\mathcal{H}^{+}$and the other is the part of $\mathcal{H}^{-}$to the right of the saddle. We will write sometimes $\mathcal{H}_{2}^{+}$and $\mathcal{H}_{2}^{-}$in order to signify them, thus $\mathcal{H}_{2}=\mathcal{H}_{2}^{+} \cap \mathcal{H}_{2}^{-}$in this case. Denote by $\Omega_{i j}$ the domain in $h<0$ which boundary is $\mathcal{H}_{i} \cup \mathcal{V}_{j}, i, j=1,2$. (More precisely in the case $B^{2}-3 A E \geq 0 \quad \Omega_{22}$ consists of two separate subdomains: $\Omega_{22}^{-}$ with boundary $\mathcal{H}_{2}^{-} \cup \mathcal{V}_{2}$ and $\Omega_{22}^{+}$with boundary $\mathcal{H}_{2}^{+} \cup\{h=0\}$; in the case $B^{2}-3 A E<0$ the boundary of $\Omega_{22}$ is $\mathcal{H}_{2} \cup \mathcal{V}_{2} \cup\{h=0\}$.)

Therefore depending on the sign of $B^{2}-3 A E$, the position of the saddle point and the availability of extrema on $\mathcal{H}^{-}$there are four cases I - IV (see Fig. 4, a,b,c,d):

I $B^{2}-3 A E<0, \mathcal{H}^{-}$has no extrema,

II $B^{2}-3 A E<0, S_{0}$ is between $S_{H}^{-}$and $\bar{S}$,

III $B^{2}-3 A E<0, S_{0}$ is between $S_{H}$ and $S_{H}^{-}$,

IV $B^{2}-3 A E \geq 0$. 
The zero isocline $\Gamma_{0}$ can be divided into two branches $\Gamma_{0}^{+}$and $\Gamma_{0}^{-}$, where $\Gamma_{0}^{ \pm}$is given by the graph of $w_{0}^{ \pm}(h), h<0$ respectively. In the case where $\mathcal{D}_{0}$ changes the sign each of $\Gamma_{0}^{ \pm}$is subdivided into the left $\left(h \leq h^{-}\right)$and right $\left(h^{+} \leq h<0\right)$ parts, thus forming again two connected components, the left one $\Gamma_{0}^{l}$ given by the union of graphs of $w_{0}^{ \pm}(h), h \leq h^{-}$and the right one $\Gamma_{0}^{r}$ given by the graphs of $w_{0}^{ \pm}(h), h^{+} \leq h<0$.

The following facts are obvious:

1) $\Gamma_{0}^{+} \subset \overline{\Omega_{21} \cup \Omega_{22}}, \quad \Gamma_{0}^{-} \subset \overline{\Omega_{11} \cup \Omega_{12}}$;

2) $w_{0}^{+}(h)$ is increasing in $\Omega_{22}$ and decreasing in $\Omega_{21}$;

3) $w_{0}^{-}(h)$ is increasing in $\Omega_{11}$ and decreasing in $\Omega_{12}$.

Finally we denote by $\mathcal{H}_{\text {min }}$ the part of $\mathcal{H}_{2}$ determined as follows:

$$
\mathcal{H}_{\text {min }}=\left\{\begin{array}{r}
\text { the part between } S_{H}^{+} \text {and } S_{0} \text { in the cases I, II, } \\
\text { the part between } S_{H}^{+} \text {and } S_{H}^{-} \text {in the case III, } \\
\text { the part of } \mathcal{H}_{2}^{+} \text {to the right of } S_{H}^{+} \text {in the case IV. }
\end{array}\right.
$$

Denote also $\mathcal{H}_{\text {max }}=\mathcal{H}_{2} \backslash \mathcal{H}_{\text {min }}$. In the cases III, IV $\mathcal{H}_{\text {max }}$ consists of two separated parts $\mathcal{H}_{\text {max }}^{ \pm} \subset \mathcal{H}^{ \pm}$.

Proof of (i). - Using the asymptotics in Lemma 4.2 (i) we see that $\Gamma_{0}^{+}$ joins the points of $\Omega_{22}(-\infty, \gamma)$ and $(-0, \gamma)$. From Lemma 3.3 (ii) it follows that $\Gamma_{0}^{+}$passes also through $\Omega_{21}$. But $\Gamma_{0}^{+}$can enter $\Omega_{21}$ only at a point of $\mathcal{H}_{\max }\left(\mathcal{H}_{\max }^{+}\right.$in cases III, IV) and can leave $\Omega_{21}$ only at a point of $\mathcal{H}_{\text {min }}$ entering again $\Omega_{22}$. After that $\Gamma_{0}^{+}$being in $\Omega_{22}$ increases up to the point $(-0, \gamma)$ as $\Gamma_{0}^{+}$cannot meet again $\mathcal{H}_{\text {max }}$. Thus $w_{0}^{+}(h), h<0$ has exactly one maximum and one minimum, which proves (i). (The very special case when $\Gamma_{0}^{+}$does form two of the saddle separatrices can be treated similarly.)

Proof of (ii). - First we note that (3.5) gives that $\left[h^{-}, h^{+}\right] \subset\left(-h_{c},-h_{s}\right)$ so we can use the inequalities from Lemma 3.3. The asymptotics in Lemma 4.2 show that the left component $\Gamma_{0}^{l}$ joins the points $(-\infty, \gamma)$ in $\Omega_{22}$ and $(-\infty,-\infty)$ in $\Omega_{11}$ while the right component of $\Gamma_{0}$ joins the point $(-0,-\infty)$ in $\Omega_{12}$ with the point $(-0, \gamma)$ in $\Omega_{22}$. Consider first the right component $\Gamma_{0}^{r}$, beginning from $(-0,-\infty)$. It can leave $\Omega_{12}$ only at a point of $\mathcal{V}_{2}$ entering $\Omega_{22}$. Using Lemma 3.3 (ii) yields that once in $\Omega_{22}, \Gamma_{0}^{r}$ must pass also through $\Omega_{21}$, which is possible only in cases III, IV. Thus after $\Gamma_{0}^{r}$ enters $\Omega_{22}$ (at a point $\left(h^{+}, w_{0}\left(h^{+}\right)\right) \in \mathcal{V}_{2}$ ) it increases up to a point of $\mathcal{H}_{\text {max }}^{-}$. Then $\Gamma_{0}^{r}$ decreases in $\Omega_{21}$ up to a point of $\mathcal{H}_{\text {min }}$. After that $\Gamma_{0}^{r}$ enters again $\Omega_{22}$ and increases up to the point $(0, \gamma)$. Thus $w_{0}^{+}(h), h^{+}<h<0$ has exactly one maximum and one minimum, which proves the case $b$ ). 
Similarly we consider the left component $\Gamma_{0}^{l}$, starting at the point $(-\infty, \gamma)$ in $\Omega_{22}$. Lemma 3.3 (ii) forces $\Gamma_{0}^{l}$ to pass successively through the domains $\Omega_{22}, \Omega_{21}, \Omega_{11}, \Omega_{12}$ and again $\Omega_{11}$. In such a way $w_{0}^{+}(h), h<h^{-}$has a unique maximum at a point of $\mathcal{H}_{\max }^{+}$and $w_{0}^{-}(h), h<h^{-}$has a unique maximum at a point on the part of $\mathcal{H}_{1}$ to the left of $S_{H}$ and a unique minimum at a point of $\mathcal{H}_{1}$ between $S_{H}$ and $S_{0}$. Lemma 4.9 is proved.

COROLlaRY 4.10. - In the case $\mathcal{D}_{0} \geq 0$ the function $w_{0}^{+}(h)$ is a monotone decreasing in $\left[-h_{c},-h_{s}\right]$. In the case when $\mathcal{D}_{0}$ changes the sign the function $w_{0}^{+}(h)$ is decreasing in $\left[-h_{c}, h^{-}\right]$and negative in $\left[h^{+},-h_{s}\right]$.

Proof. - The monotony of $w_{0}^{+}(h)$ we claim follows immediately from Lemmas 3.3 and 4.9 using also the behaviour of $w_{0}^{+}$near $-\infty$ and -0 . Further, the same argument shows that the minimum of $w_{0}^{-}(h)$ is negative. On the other hand the maximum of $\Gamma_{0}^{r}$ is situated on $\mathcal{H}_{\text {max }}^{-}$and hence below the saddle. The minimum of $\Gamma_{0}^{l}$ is situated on the arc between $S_{H}$ and $S_{0}$ above the saddle. Therefore the maximum of $w_{0}^{+}(h)$ is also negative which proves the corollary.

\section{REGULARITY OF THE CENTROID CURVE}

We intend to prove the regularity of the curve $L$ in the general (not necessarily symmetric) case of generic Hamiltonian systems with two saddles and two centres. In this case the Hamiltonian in an appropriate coordinate system has a normal form

$$
H=\frac{x^{2}+y^{2}}{2}+A x^{3}+B x^{2} y+C x y^{2}, \quad B^{2}-4 A C<0 .
$$

The critical values of $H$ are $0=h_{1}<h_{2}<h_{3}<h_{4}$, where $h_{1}$ and $h_{4}$ correspond to the centres and $h_{2}, h_{3}$ to the saddles. As in [16] we shall prove a stronger result than regularity:

THEOREM 5.1. - There exists a linear combination of the integrals $X, Y$ for which the corresponding ratio $(r X+s Y) / M$ is a monotone function, i.e. $r \xi^{\prime}(h)+s \eta^{\prime}(h) \neq 0$ for $h \in\left[h_{1}, h_{2}\right)$.

Theorem 5.1 easily follows from the next one:

THEOREM 5.1'. - There exist real numbers $r, s$ such that for any real $k$ the function $r X^{\prime}+s Y^{\prime}-k M^{\prime}$ has no more than one zero in $\left[h_{1}, h_{2}\right)$.

Assuming that Theorem $5.1^{\prime}$ is proved we get that the function $r X+s Y-k M$ has no more than two zeros, one of them being $h_{1}$, 
which shows that $(r X+s Y) / M-k$ has for any $k$ at most one zero, i.e. $(r X+s Y) / M$ is a monotone function.

For the proof of Theorem 5.1', we intend to use an adaptation of Petrov's method [22]. For this reason we need to introduce some notation and recall some notions and results from Picard-Lefschetz theory (see [3] for details). The equation of the level-curves $\Gamma_{h}:\{H=h\}$ can be rewritten in the form $\left[(1+2 C x) y+B x^{2}\right]^{2}=\left(B^{2}-4 A C\right) x^{4}-2(A+C) x^{3}-x^{2}+4 C h x+2 h$, which motivates the definition of the following forms [12]:

$$
\begin{aligned}
\omega_{X} & =\frac{x d x}{(1+2 C x) y+B x^{2}}, \quad \omega_{Y}=\frac{y d x}{(1+2 C x) y+B x^{2}}, \\
\omega_{M} & =\frac{d x}{(1+2 C x) y+B x^{2}} .
\end{aligned}
$$

Obviously

$$
X^{\prime}=-\int_{\delta(h)} \omega_{X}, \quad Y^{\prime}=-\int_{\delta(h)} \omega_{Y}, \quad M^{\prime}=-\int_{\delta(h)} \omega_{M} .
$$

For later use put also $\omega=\alpha \omega_{X}+\beta \omega_{Y}+\gamma \omega_{M}$. In this section we are going to work with the complexification of the family of curves $\Gamma_{h}$, i. e. we define

$$
\Gamma_{h}^{\mathbb{C}}=\left\{(x, y) \in \mathbb{C}^{2}: H(x, y)=h, h \in \mathbb{C}\right\} .
$$

Thus $\Gamma_{h}^{\mathbb{C}}$ has a topological type of a torus, punctured at three points $P_{1}, P_{2}, P_{3}$. For $j=1, \ldots 4$ we define elements $\alpha_{j}$ in the fundamental group $\pi_{1}\left(\mathbb{C} \backslash\left\{h_{1}, h_{2}, h_{3}, h_{4}\right\}\right)$, so that $\alpha_{j}$ is represented by a small circle around $h_{j}$ oriented counter-clockwise. Also we define a vanishing cycle $\delta_{j}(h)$ corresponding to $\alpha_{j}$. The cycles $\delta_{j}(h)$ generate the first homology group $H_{1}\left(\Gamma_{h}^{\mathbb{C}}\right)$. For further use we denote by $M_{k}$ the monodromy operators corresponding to $\alpha_{k} \in \pi_{1}$. Given a function $f_{\delta_{i} \delta_{j}}$ depending on $\delta_{i}, \delta_{j} \in H_{1}\left(\Gamma_{h}^{\mathbb{C}}\right)$ we define by $M_{k}^{*} f_{\delta_{i} \delta_{j}}$ the function $f_{M_{k} \delta_{i} M_{k} \delta_{j}}$.

Denote by $\bar{\Gamma}_{h}$ the smooth compactification of $\Gamma_{h}^{\mathbb{C}}$. We define $\delta_{1}(h)$ for $h \in\left(h_{1}, h_{2}\right)$ to be represented by the compact real component (the oval) of $\Gamma_{h}^{\complement}$ oriented counter-clockwise. Similarly we define $\delta_{4}(h)$ for $h \in\left(h_{3}, h_{4}\right)$ to be the compact real component of $\Gamma_{h}^{C}$. We also choose $\delta_{3}$ to be homological to $\delta_{2}$ on the compactification of $\Gamma_{h}^{\mathrm{C}}$. The orientation on $\delta_{2}$ will be such that $\left(\delta_{1} \circ \delta_{2}\right)=1$ where $(\gamma \circ \delta)$ denotes the intersection number of the cycles $\gamma, \delta \in H_{1}\left(\Gamma_{h}^{\mathbb{C}}\right)$.

As we are going to use the Picard-Lefschetz theory we need the intersection numbers between the cycles $\delta_{j}$. We formulate the result in the following lemma: 
LEMMA 5.2. - The intersection numbers of the cycles $\delta_{j}$ are given by the matrix

$$
\mathcal{A}=\left(\begin{array}{cccc}
0 & +1 & +1 & +1 \\
-1 & 0 & 0 & -1 \\
-1 & 0 & 0 & -1 \\
-1 & +1 & +1 & 0
\end{array}\right)
$$

where $\mathcal{A}_{i j}=\left(\delta_{i} \circ \delta_{j}\right)$.

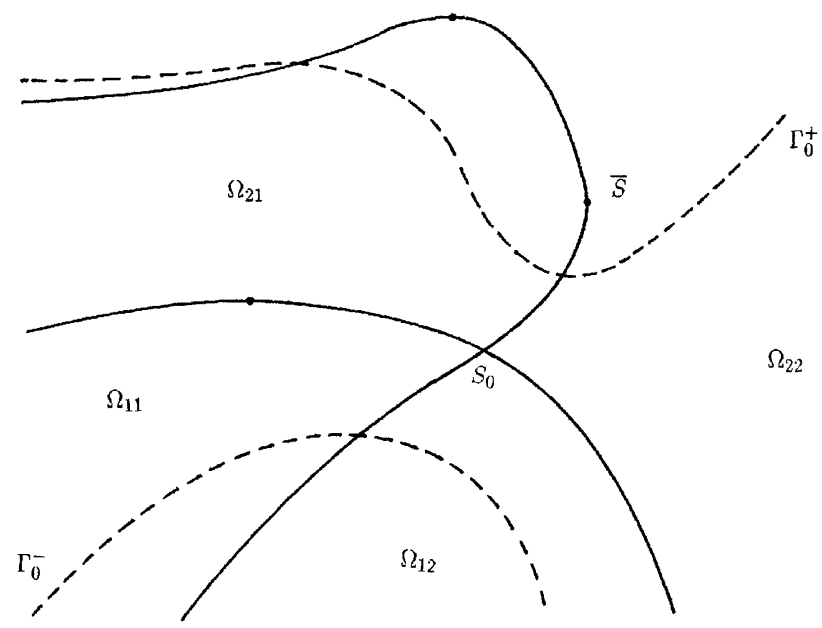

(a)

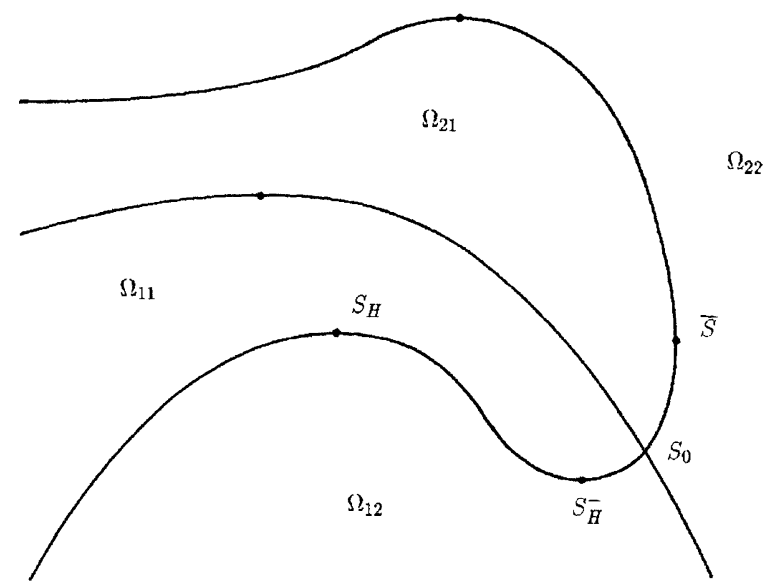

Fig. 4. 

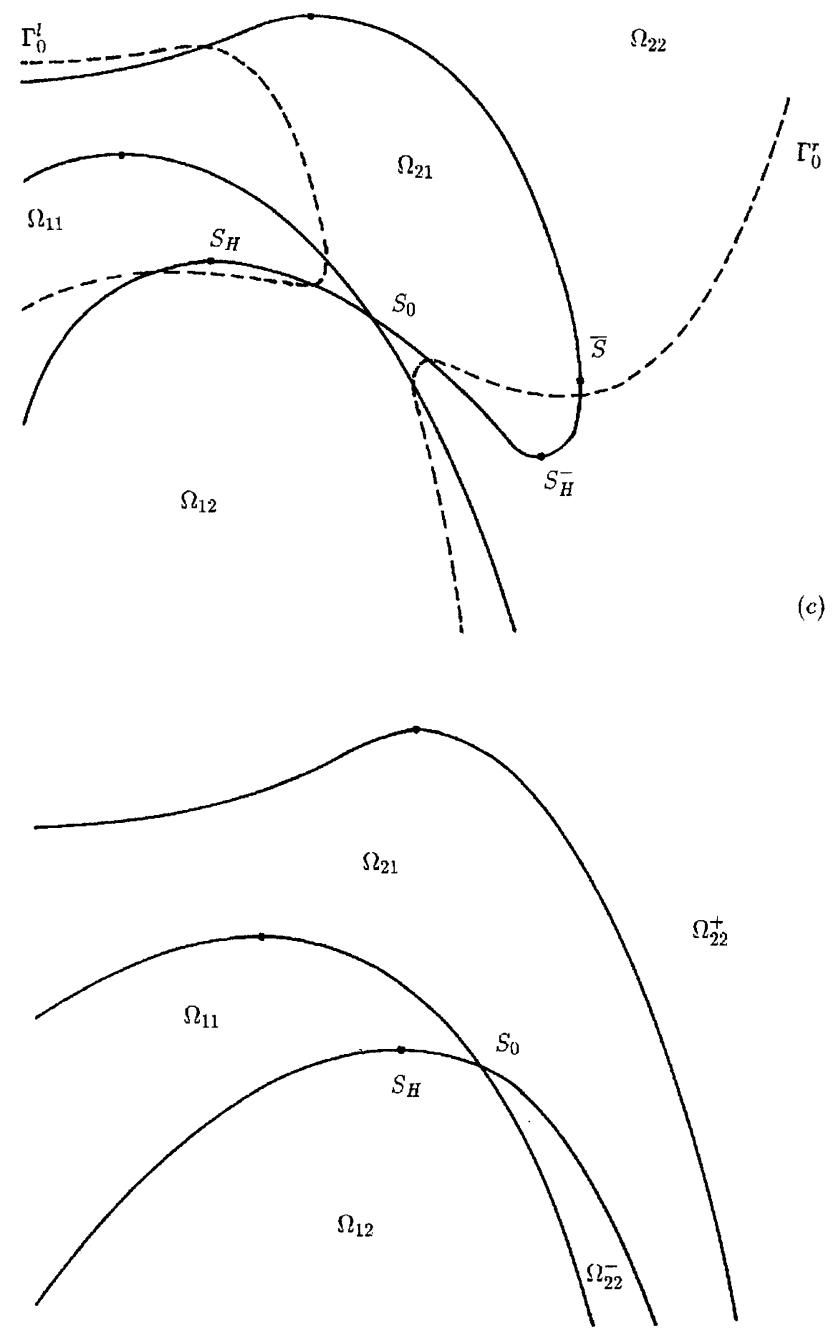

(c)

Fig. 4 continued.

Proof. - The choice of the cycles shows that we have to compute $\left(\delta_{1} \circ \delta_{4}\right)$ and $\left(\delta_{2} \circ \delta_{4}\right)=\left(\delta_{3} \circ \delta_{4}\right)$ as $\left(\delta_{1} \circ \delta_{2}\right)=\left(\delta_{1} \circ \delta_{3}\right)=1,\left(\delta_{2} \circ \delta_{3}\right)=0$. Obviously $\delta_{1}$ and $\delta_{2}$ are generators of $H_{1}\left(\bar{\Gamma}_{h}\right)$ hence we have $\delta_{4}=n_{1} \delta_{1}+n_{2} \delta_{2}$. Then

$$
\left(\delta_{4} \circ \delta_{1}\right)=n_{2}\left(\delta_{2} \circ \delta_{1}\right)=-n_{2}, \quad\left(\delta_{4} \circ \delta_{2}\right)=n_{1}\left(\delta_{1} \circ \delta_{2}\right)=n_{1},
$$

Define the functions

$$
J_{M, j}(h)=\int_{\delta_{j}(h)} \omega_{M}, \quad h \in\left(h_{1}, h_{2}\right) .
$$


Our idea to compute the numbers $n_{1}$ and $n_{2}$ is to use Picard-Lefschetz formula and the reality of $J_{M, 1}$ on $\left(h_{1}, h_{2}\right)$ and of $J_{M, 4}$ on $\left(h_{3}, h_{4}\right)$. Define a path $S$ on the punctured plane $\mathbb{C} \backslash\left\{h_{1}, h_{2}, h_{3}, h_{4}\right\}$ in the following way. Take a point $h^{\prime} \in\left(h_{1}, h_{2}\right)$ and a point $h^{\prime \prime} \in\left(h_{3}, h_{4}\right)$ and connect them with a smooth path

$$
S^{+}=\left\{h(s), s \in[0,1], h(0)=h^{\prime}, h(1)=h^{\prime \prime}, \operatorname{Im} h(s)>0, s \in(0,1)\right\} .
$$

Put $S=S^{+} \cup \overline{S^{+}}$, oriented counter-clockwise (see Fig. 5). Then PicardLefschetz formula yields

$$
S_{*} \delta_{1}=\delta_{1}+\left(\delta_{2} \circ \delta_{1}\right) \delta_{2}+\left(\delta_{3} \circ \delta_{1}\right) \delta_{3} .
$$

Denote by $J_{M, 1}^{+}$the continuation of $J_{M, 1}$ along $S^{+}$and put $J_{M, 1}^{+}(h)=$ $\lambda+i \mu$ with $\lambda, \mu$ real. Then for $h \in \overline{S^{+}}$we have:

$$
J_{M, 1}^{+}(h)=\overline{J_{M, 1}^{+}}(\bar{h}), \quad \bar{h} \in S^{+} .
$$

Then starting from $h^{\prime \prime} \in\left(h_{3}, h_{4}\right)$ and making one turn along $S$ the function $J_{M, 1}(h)$ will change by

$$
-\int_{\delta_{2}} \omega_{M}-\int_{\delta_{3}} \omega_{M}=-2 \int_{\delta_{2}} \omega_{M}
$$

according to (5.1). This means that

$$
-2 \int_{\delta_{2}} \omega_{M}=J_{M, 1}^{+}\left(h^{\prime \prime}\right)-J_{M, 1}^{-}\left(h^{\prime \prime}\right)=2 i \mu, \text { i.e. } \int_{\delta_{2}} \omega_{M}=-i \mu
$$

Thus we obtain

$$
\int_{\delta_{1}} \omega_{M}=J_{M, 1}^{+}=\lambda+i \mu=\lambda-\int_{\delta_{2}} \omega_{M}, \quad h \in\left(h_{3}, h_{4}\right) .
$$

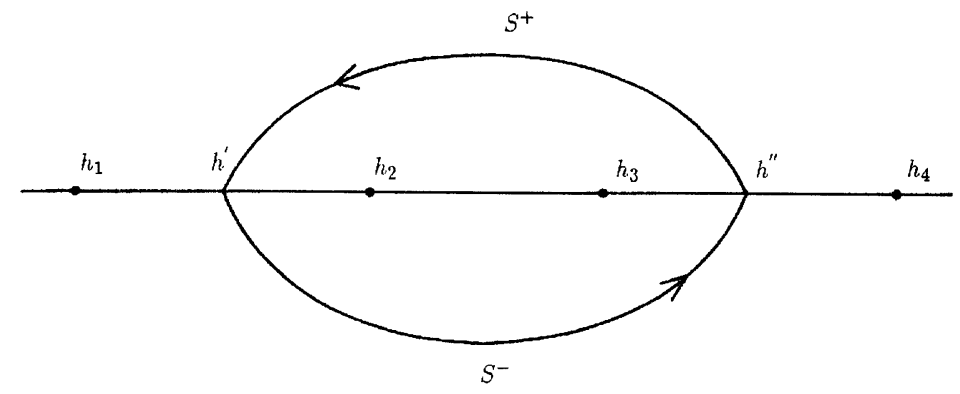

Fig. 5. 
Hence

$$
\begin{aligned}
\int_{\delta_{4}} \omega_{M} & =n_{1} \int_{\delta_{1}} \omega_{M}+n_{2} \int_{\delta_{2}} \omega_{M} \\
& =n_{1} \lambda+\left(n_{2}-n_{1}\right) \int_{\delta_{2}} \omega_{M}=n_{1} \lambda-\left(n_{2}-n_{1}\right) i \mu
\end{aligned}
$$

Now notice that $\int_{\delta_{4}(h)} \omega_{M}$ is real on $\left(h_{3}, h_{4}\right)$. This shows that $n_{2}-n_{1}=0$, i.e. $\delta_{4} \sim n_{1}\left(\delta_{1}+\delta_{2}\right)$. On the other hand $\delta_{3}, \delta_{4}$ can be chosen as generators of $H_{1}\left(\overline{\Gamma_{h}^{\mathbb{C}}}\right)$ which gives $n_{1}= \pm 1$. We choose the orientation of $\delta_{4}$ so that $n_{1}=1$.

Remark. - There is a general method proposed by S. M. Gusein-Zade [13], [14] and N. A'Campo [1] for computing the intersection matrix for the level lines of functions of two variables (see also [3], §4). In order to apply this method we need to compute the intersection matrix only for the Hamiltonian (0.3) with $\mu=0$. We prefer however the independent proof stated above for the following reasons. First, our proof is in the spirit of rest of this section. Second, we introduce explicitly the vanishing cycles $\delta_{j}$ which we will use later.

Although $\delta_{4}$ and $\delta_{1}+\delta_{2}$ represent the same class in $H_{1}\left(\overline{\Gamma^{\mathbb{C}}}\right) \simeq \mathbb{Z}^{2}$, they represent different elements in $H_{1}\left(\Gamma^{\mathbb{C}}\right) \simeq \mathbb{Z}^{4}$. The same is true for $\delta_{2}, \delta_{3}$. Denote by $\rho_{1}$ the element $\left[\delta_{4}-\delta_{1}-\delta_{2}\right] \in H_{1}\left(\Gamma^{\mathrm{C}}\right)$ and by $\rho_{2}-$ the element $\left[\delta_{2}-\delta_{3}\right] \in H_{1}\left(\Gamma^{\mathbb{C}}\right)$. Obviously $\rho_{1}, \rho_{2}$ can be represented by unions of small circles around $P_{1}, P_{2}, P_{3}$. Following [22], [12] we define the function

$$
F(h)=\frac{I^{\prime}(h)}{M^{\prime}(h)}, \quad h \in\left[h_{1}, h_{2}\right),
$$

where $I^{\prime}(h)=\alpha X^{\prime}+\beta Y^{\prime}+\gamma M^{\prime}$. The function $F(h)$ can be continued analytically for any $h \in \mathbb{C} \backslash\left\{h_{2}, h_{3}, h_{4}\right\}$ but the continuation depends on the path. We can consider the domain $\mathcal{D}=\mathbb{C} \backslash\left\{h \geq h_{2}\right\}$ where $F$ can be defined as univalent function.

For any function $f(h)$ and $h \in \mathcal{D}$ we denote by $f^{+}\left(h_{0}\right) \quad\left(f^{-}\left(h_{0}\right)\right), \quad \operatorname{Im} h_{0}=0$ the result of the analytic continuation of $f(h)$ along a path with $\operatorname{Im} h>0 \quad(\operatorname{Im} h<0)$.

In the rest of this section we will need the Wronskians

$$
W_{\delta_{i}, \delta_{j}}(h)=\left|\begin{array}{c}
\int_{\delta_{i}(h)} \omega_{M} \int_{\delta_{j}(h)} \omega_{M} \\
\int_{\delta_{i}(h)} \omega \int_{\delta_{j}(h)} \omega
\end{array}\right|
$$


LEMMA 5.3. - On the intervals $\left(h_{2}, h_{3}\right),\left(h_{3}, h_{4}\right),\left(h_{4}, \infty\right)$ the imaginary part of $F$ has the representation

$$
\operatorname{Im} F^{ \pm}(h)=\left\{\begin{array}{l} 
\pm \frac{i}{2\left|J_{\omega_{M}}\right|^{2}} W_{\delta_{1}, \delta_{2}}(h), \quad h \in\left(h_{2}, h_{3}\right) \\
\pm \frac{i}{2\left|J_{\omega_{M}}\right|^{2}} W_{\delta_{1}, \delta_{2}+\delta_{3}}(h), \quad h \in\left(h_{3}, h_{4}\right), \\
\pm \frac{i}{2 \mid J_{\left.\omega_{M}\right|^{2}}} W_{\delta_{1}, \delta_{2}+\delta_{3}-3 \delta_{4}}(h), \quad h \in\left(h_{4}, \infty\right) .
\end{array}\right.
$$

The proof essentially repeats [12] using Lemma 5.2. Denote

$$
\begin{aligned}
& G_{1}(h)=2\left|J_{\omega_{M}}\right|^{2} \operatorname{Im} F^{+}(h) \text { in }\left(h_{2}, h_{3}\right), \\
& G_{2}(h)=2\left|J_{\omega_{M}}\right|^{2} \operatorname{Im} F^{+}(h) \text { in }\left(h_{3}, h_{4}\right), \\
& G_{3}(h)=2\left|J_{\omega_{M}}\right|^{2} \operatorname{Im} F^{+}(h) \text { in }\left(h_{4}, \infty\right) .
\end{aligned}
$$

Define also the complex domains $\mathcal{D}_{1}=\mathbb{C} \backslash\left\{\left[h_{3}, \infty\right)\right\}, \quad \mathcal{D}_{2}=\mathbb{C} \backslash$ $\left\{\left[h_{2}, h_{3}\right] \cup\left[h_{4}, \infty\right)\right\}, \mathcal{D}_{3}=\mathbb{C} \backslash\left\{\left[h_{2}, h_{4}\right]\right\}$ (see Fig. 6).

LEMMA 5.4. - The functions $G_{1}, G_{2}, G_{3}$ are holomorphic and singlevalued respectively in $\mathcal{D}_{1}, \mathcal{D}_{2}, \mathcal{D}_{3}$.

The proof repeats the proof of Lemma 4.4 from [12].
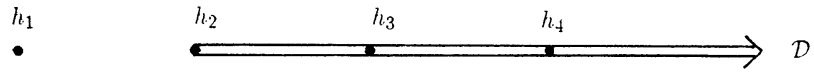

$h_{1}$

$$
h_{2}
$$

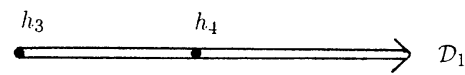

$h_{1}$
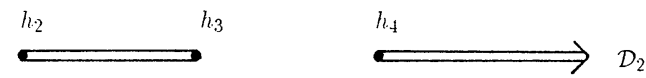

$h_{1}$

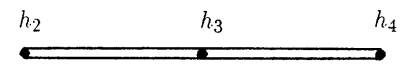

$\mathcal{D}_{3}$

Fig. 6. 
LEMMA 5.5. - The following formulas are true:

$$
\begin{aligned}
& \operatorname{Im} G_{1}^{ \pm}(h)= \pm\left\{\begin{array}{l}
-W_{\delta_{3}, \delta_{2}}(h), h \in\left(h_{3}, h_{4}\right) \\
-W_{\delta_{3}, \delta_{2}}(h)+W_{\delta_{1}-\delta_{3}+2 \delta_{2}, \delta_{4}}(h), \quad h \in\left(h_{4}, \infty\right),
\end{array}\right. \\
& \operatorname{Im} G_{2}^{ \pm}(h)= \pm \begin{cases}W_{\delta_{3}, \delta_{2}}(h), \quad h \in\left(h_{2}, h_{3}\right) \\
-W_{\delta_{4}, \delta_{2}+\delta_{3}+2 \delta_{1}}(h), \quad h \in\left(h_{4}, \infty\right),\end{cases} \\
& \operatorname{Im} G_{3}^{ \pm}(h)= \pm\left\{\begin{array}{l}
W_{2 \delta_{4}, \delta_{1}+\delta_{3}}(h)-W_{\delta_{1}, \delta_{3}-\delta_{2}}(h)+W_{\delta_{4}-\delta_{1}, \delta_{2}}(h), \\
h \in\left(h_{2}, h_{3}\right) \\
-W_{\delta_{4}, \delta_{2}+\delta_{3}+2 \delta_{1}}(h),
\end{array}\right.
\end{aligned}
$$

Proof. - We will write $G_{j}$ without the superscripts " \pm ".

(i). Take a point $h_{0} \in\left(h_{3}, h_{4}\right)$. Then Picard-Lefschetz formula yields

$$
\operatorname{Im} G_{1}\left(h_{0}\right)=\left(W_{\delta_{1}, \delta_{2}}^{-}-W_{\delta_{1}, \delta_{2}}^{+}\right)\left(h_{0}\right)=M_{3}^{*}\left(W_{\delta_{1}, \delta_{2}}\right)-W_{\delta_{1}, \delta_{2}} .
$$

Using that $W_{\gamma, \delta}$ is bilinear and antisymmetric in $\gamma$ and $\delta$ and that $M_{3}^{*}\left(\delta_{1}\right)=\delta_{1}-\delta_{3}, M_{3}^{*}\left(\delta_{2}\right)=\delta_{2}$ (Lemma 5.2), we get $M_{3}^{*}\left(W_{\delta_{1}, \delta_{2}}\right)\left(h_{0}\right)-$ $W_{\delta_{1}, \delta_{2}}\left(h_{0}\right)=-W_{\delta_{3}, \delta_{2}}\left(h_{0}\right)$. In the same manner for $h_{0} \in\left(h_{4}, \infty\right)$ we have $\operatorname{Im} G_{1}\left(h_{0}\right)=M_{3}^{*} M_{4}^{*}\left(W_{\delta_{1}, \delta_{2}}\right)\left(h_{0}\right)-W_{\delta_{1}, \delta_{2}}$ using that

$$
\left(M_{3} M_{4}\right)^{*}\left(\delta_{1}\right)=\delta_{1}-\delta_{3}-2 \delta_{4}, \quad\left(M_{3} M_{4}\right)^{*}\left(\delta_{2}\right)=\delta_{2}+\delta_{4} .
$$

Hence $\left(M_{3} M_{4}\right)^{*} W_{\delta_{1}, \delta_{2}}-W_{\delta_{1}, \delta_{2}}=-W_{\delta_{3}, \delta_{2}}+W_{\delta_{1}-\delta_{3}+2 \delta_{2}, \delta_{4}}$.

(ii). Consider $\operatorname{Im} G_{2}$ on the interval $\left(h_{4}, \infty\right)$. The above argument gives

$$
\operatorname{Im} G_{2}=M_{4}^{*}\left(W_{\delta_{1}, \delta_{2}+\delta_{3}}\right)-W_{\delta_{1}, \delta_{+} \delta_{3}}=-W_{\delta_{4}, \delta_{2}+\delta_{3}+2 \delta_{1}} .
$$

To compute $\operatorname{Im} G_{2}$ on the interval $\left(h_{2}, h_{3}\right)$ we need to act on $W_{\delta_{1}, \delta_{2}+\delta_{3}}$ by $\left(M_{3}^{-1}\right)^{*}$ which gives $\operatorname{Im} G_{2}=W_{\delta_{3}, \delta_{2}}$.

(iii). Repeating the above argument we obtain for $h \in\left(h_{3}, h_{4}\right)$

$$
\operatorname{Im} G_{3}=\left(M_{4}\right)^{*} W_{\delta_{1}, \delta_{2}+\delta_{3}+\delta_{4}}-W_{\delta_{1}, \delta_{2}+\delta_{3}+\delta_{4}}=-W_{\delta_{4}, \delta_{2}+\delta_{3}+2 \delta_{1}} .
$$

When $h \in\left(h_{2}, h_{3}\right)$ we have

$$
\begin{aligned}
\operatorname{Im} G_{3} & =\left(M_{4} M_{3}\right)^{*} W_{\delta_{1}, \delta_{2}+\delta_{3}-3 \delta_{4}}-W_{\delta_{1}, \delta_{2}+\delta_{3}-3 \delta_{4}} \\
& =W_{2 \delta_{4}, \delta_{1}+\delta_{3}}-W_{\delta_{1}, \delta_{3}-\delta_{2}}+W_{\delta_{4}-\delta_{1}, \delta_{2}} .
\end{aligned}
$$


Lemma 5.5 is proved.

$$
\begin{aligned}
& \text { Corollary 5.6. }- \text { Denote } r_{1}=\int_{\rho_{1}} \omega, r_{2}=\int_{\rho_{2}} \omega . \text { Then } \\
& \operatorname{Im} G_{1}^{ \pm}(h)= \pm\left\{\begin{array}{l}
r_{2} \int_{\delta_{3}} \omega_{M}, \quad h \in\left(h_{3}, h_{4}\right) \\
\left(r_{2}+r_{1}\right) \int_{\delta_{4}} \omega_{M}+r_{2} \int_{\delta_{3}} \omega_{M}, \quad h \in\left(h_{4}, \infty\right),
\end{array}\right. \\
& \operatorname{Im} G_{2}^{ \pm}(h)= \pm\left\{\begin{array}{l}
-r_{2} \int_{\delta_{3}} \omega_{M}, \quad h \in\left(h_{2}, h_{3}\right) \\
-\left(r_{2}+2 r_{1}\right) \int_{\delta_{4}} \omega_{M}, \quad h \in\left(h_{4}, \infty\right), \\
\operatorname{Im} G_{3}^{ \pm}(h)= \pm\left\{\begin{array}{l}
-\left(r_{2}+r_{1}\right) \int_{\delta_{3}} \omega_{M}-\left(r_{2}+2 r_{1}\right) \int_{\delta_{4}} \omega_{M}, \\
-\left(r_{2}+2 r_{1}\right) \int_{\delta_{4}} \omega_{M}, \quad h \in\left(h_{3}, h_{4}\right) .
\end{array}\right.
\end{array}\right.
\end{aligned}
$$

COROLlaRY 5.7. - The constant $r_{2}$ is purely imaginary. The constant $r_{2}+2 r_{1}$ is real.

Proof. - The integral $\int_{\delta_{3}} \omega_{M}$ is purely imaginary on $\left(h_{2}, h_{4}\right)$. The integral $\int_{\delta_{4}} \omega_{M}$ is real on $\left(h_{3}, \infty\right)$.

Now we choose the constants $\alpha$ and $\beta$ in (5.2) so that $r_{2} \neq 0$, $r_{2}+2 r_{1}=0$. To specify the above construction for this special case we need the domains

$$
\mathcal{D}_{01}=\mathcal{D}_{1}, \quad \mathcal{D}_{02}=\mathcal{D}_{03}=\mathbb{C} \backslash\left\{\left[h_{2}, h_{3}\right]\right\} .
$$

Denote by $F_{0}, G_{0 i}$ the corresponding functions $F, G_{i}$ in this case.

LEMMA 5.8. - (i) The functions $G_{0 i}, \quad i=1,2,3$ are holomorphic and single-valued respectively in the domains $\mathcal{D}_{0 i}$.

(ii) The imaginary parts satisfy:

$$
\operatorname{Im} G_{01}^{ \pm}(h)= \pm\left\{\begin{array}{l}
r_{2} \int_{\delta_{3}} \omega_{M}, \quad h \in\left(h_{3}, h_{4}\right) \\
r_{2} \int_{\delta_{3}} \omega_{M}+\frac{1}{2} r_{2} \int_{\delta_{4}} \omega_{M}, \quad h \in\left(h_{4}, \infty\right),
\end{array}\right.
$$


PERTURBATIONS OF QUADRATIC HAMILTONIAN SYSTEMS WITH SYMMETRY

$$
\begin{array}{cc}
\operatorname{Im} G_{02}^{ \pm}(h)=\mp r_{2} \int_{\delta_{3}} \omega_{M}, & h \in\left(h_{2}, h_{3}\right), \\
\operatorname{Im} G_{03}^{ \pm}(h)=\mp \frac{1}{2} r_{2} \int_{\delta_{3}} \omega_{M}, & h \in\left(h_{2}, h_{3}\right) .
\end{array}
$$

LEMMA 5.9. - The functions $G_{0 i}, i=1,2,3$ have no zeros in the corresponding domains $\mathcal{D}_{0 i}$.

Proof. - Consider the function $G_{01}$. Let $R$ be a big enough constant and $\varrho$ is a sufficiently small number. Denote by $\mathcal{D}_{01}^{0}$ the domain obtained from $\mathcal{D}_{01} \cap\{|h|<R\}$ by removing circles of radius $\varrho$ around $h_{3}$ and $h_{4}$ (see Fig. 7). Lemma 4.6 from [12] gives that along the circle $\{|h|=R\}$ the argument of $G_{01}$ increases by no more than $-2 \pi / 3$ (i.e. decreases by at least $2 \pi / 3)$. Near the point $h_{4}$ the function $G_{01}(h)$ has an expansion of the type $\ln \left|h-h_{4}\right| \cdot g_{1}(h)+g_{2}(h)$, with some holomorphic $g_{i}(h)$. Hence the increase of the argument of $G_{01}$ along the circle $\left|h-h_{4}\right|=\varrho$ can be made arbitrarily small. On the intervals $\left(h_{3}+\varrho, h_{4}-\varrho\right)$ and $\left(h_{4}+\varrho, \infty\right)$ the function $\operatorname{Im} G_{01}$ has no zeros as it is represented by a nonzero multiple of the real period of $\omega_{M}$. Along the circle $\left|h-h_{3}\right|=\varrho$ the function $\operatorname{Im} G_{01}$ has exactly one zero. This shows that running the boundary of $\mathcal{D}_{01}^{0}$ the argument of $G_{01}$ increases by no more than $2 \pi-2 \pi / 3$, hence it does not increase. The argument principle yields that $G_{01}$ has no zeros in $\mathcal{D}_{01}$.

The functions $G_{02}$ and $G_{03}$ are treated along the same lines, introducing the corresponding domains $\mathcal{D}_{02}^{0}=\mathcal{D}_{03}^{0}$ (see Fig. 7). Now the proof of Theorem $5.1^{\prime}$ repeats literally that of Theorem $4.1^{\prime}$ of [16].

This proves the regularity of $L$ for the case of generic Hamiltonians with both two centres and saddles.
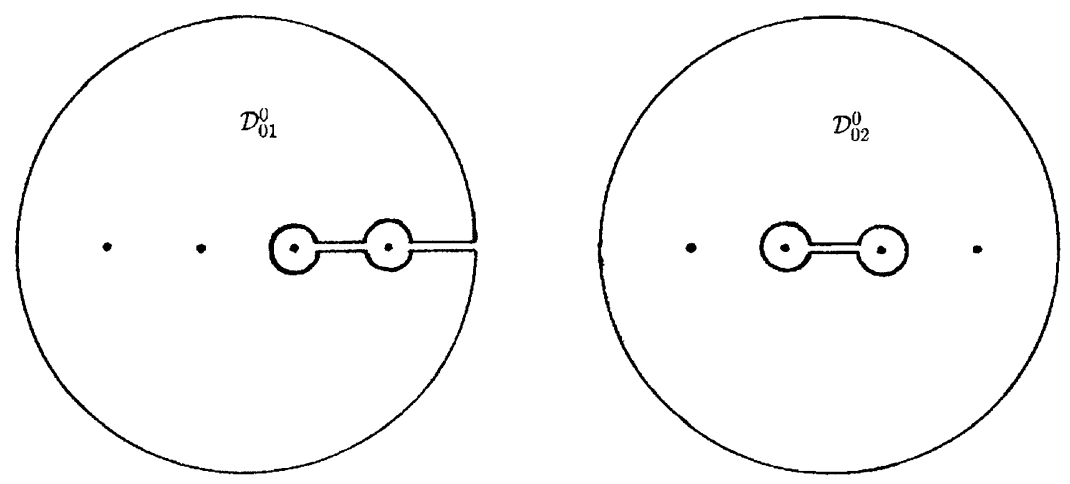

Fig. 7. 


\section{THE CENTROID CURVE OF THE PERTURBED STANDARD HAMILTONIAN}

As we intend to apply the deformation argument described in section 1, we start with the standard elliptic Hamiltonian (given in (3.3) by $\lambda=0$ ) for which the centroid curve is a line segment. For small $\lambda>0$ we can use results from [9] to show that $L$ is strictly convex.

In [16] we have derived Picard-Fuchs system of equations for the functions $X, Y, M$ and the auxiliary function $K=\iint_{\text {Int } \delta(h)} x y d x d y$ (see [16], formula (3.5)). We recall these equations for the special case we need, putting $a=0$ and $b=\lambda$ :

$$
\left\{\begin{array}{l}
\lambda X^{\prime}-Y^{\prime}-6 \lambda h M+4 \lambda M=0 \\
\left(1-6 \lambda^{2} h\right) Y^{\prime}+\lambda^{2} K^{\prime}+6 \lambda^{2} Y+\lambda M=0 \\
\lambda(6 h-1) X^{\prime}+K^{\prime}-6 \lambda X+\lambda M=0 \\
{\left[(1-6 h) \lambda^{2}+1\right] K^{\prime}+\lambda X+8 \lambda^{2} K-\lambda^{2} Y=0}
\end{array}\right.
$$

The Hamiltonian (3.3) is invariant under the change of the variables $(x, y, \lambda) \rightarrow(x,-y,-\lambda)$. This implies that $M$ and $X$ as functions of $\lambda$ are even functions and $Y$ and $K$ are odd ones. Hence we can write

$$
\begin{aligned}
M & =M_{0}+\frac{1}{2} \lambda^{2} M_{1}+\ldots \\
X & =X_{0}+\frac{1}{2} \lambda^{2} X_{1}+\ldots \\
Y & =\lambda Y_{0}+\frac{1}{6} \lambda^{3} Y_{1}+\ldots \\
K & =\lambda K_{0}+\frac{1}{6} \lambda^{3} K_{1}+\ldots
\end{aligned}
$$

Dividing (6.1) by $\lambda$ and putting $\lambda=0$, we get a system for the first terms in (6.2)

$$
\begin{aligned}
& X_{0}^{\prime}-Y_{0}^{\prime}-6 h M_{0}^{\prime}+4 M_{0}=0 \\
& Y_{0}^{\prime}+M_{0}=0 \\
& (6 h-1) X_{0}^{\prime}+K_{0}^{\prime}-6 X_{0}+M_{0}=0 \\
& K_{0}^{\prime}+X_{0}=0 .
\end{aligned}
$$

Eliminating $K_{0}^{\prime}$ and $Y_{0}^{\prime}$ from (6.3) we obtain Picard-Fuchs system for $M_{0}, X_{0}$ :

$$
\begin{aligned}
& 6 h(6 h-1) M_{0}^{\prime}=6(5 h-1) M_{0}+7 X_{0} \\
& (6 h-1) X_{0}^{\prime}=-M_{0}+7 X_{0},
\end{aligned}
$$


which implies Riccati equation for $\xi_{0}(h)=X_{0}(h) / M_{0}(h)$ :

$$
6 h(6 h-1) \xi_{0}^{\prime}=-6 h+6(2 h+1) \xi_{0}-7 \xi_{0}^{2} .
$$

Now paraphrasing Lemma 3.4 from [9] we get

LEMma 6.1. - (i) For $h \in\left[0, \frac{1}{6}\right)$ we have $\xi_{0}^{\prime} \geq \frac{1}{2}$ and $\xi_{0}^{\prime \prime}>0$.

(ii) When $h \rightarrow \frac{1}{6}$, we have $\xi_{0}^{\prime} \rightarrow+\infty$ and $\xi_{0}^{\prime \prime} \rightarrow+\infty$.

The direct proof is based on (6.4), but in fact the statement follows immediately from [9] noticing that our $\xi_{0}(h)$ equals $1+R\left(\frac{1}{6}-h\right)$ in the notation there. Now multiply the second equation in (6.3) by -10 and add it to the first one. We get $X_{0}^{\prime}-11 Y_{0}^{\prime}-\left(6 h M_{0}\right)^{\prime}=0$. Integrating the last equation and using that $X_{0}(0)=Y_{0}(0)=M_{0}(0)=0$, we obtain

$$
Y_{0}=\frac{1}{11} X_{0}-\frac{6 h}{11} M_{0} .
$$

Hence using (6.2) for small enough $\lambda$ we get the asymptotics

$$
\begin{aligned}
& \xi(h)=\xi_{0}(h)+O\left(\lambda^{2}\right) \\
& \eta(h)=\frac{1}{11} \lambda \xi_{0}(h)-\frac{6}{11} \lambda h+O\left(\lambda^{3}\right),
\end{aligned}
$$

which implies for the curvature $\kappa$ of $L$ at $(\xi(h), \eta(h)), h \in\left(0, \frac{1}{6}\right)$ :

$$
\kappa=\Delta(h)\left[\left(\xi^{\prime}(h)\right)^{2}+\left(\eta^{\prime}(h)\right)^{2}\right]^{-3 / 2}>0
$$

as

$$
\Delta(h)=\xi^{\prime}(h) \eta^{\prime \prime}(h)-\xi^{\prime \prime}(h) \eta^{\prime}(h)=\frac{6}{11} \lambda \xi_{0}^{\prime \prime}(h)+O\left(\lambda^{3}\right)>0 .
$$

Returning to the initial normal form (0.3) we obtain the following theorem

THEOREM 6.2. - For $\mu$ close enough to 1 the centroid curves are strictly convex.

\section{GLOBAL PHASE PORTRAITS}

In this section we prove our main Theorem 1 . Before that we recall $\ell$ is the line on which the divergence in $(0.1)$ vanishes. The corresponding equation reads $-\left(f_{x}+g_{y}\right) \equiv \alpha x+\beta y+\gamma=0$. We begin with the following simple consequence of Theorem 5.1. 
PROPOSITION 7.1. - When running $L_{1}$, the tangential vector rotates within an angle less than $\pi$.

Proof. - Given $h$, denote by $\bar{\ell}$ the tangential vector at the corresponding point of $L_{1}: \bar{\ell}=\left(\xi^{\prime}(h), \eta^{\prime}(h)\right)$. From Theorem 5.1 it follows the existence of a vector $\bar{\rho}=(r, s)$ with the property that the inner product of $\bar{\rho}$ and $\bar{\ell}$ is non-zero for all $h$. Therefore $\bar{\ell}$ deviates with respect to $\bar{\rho}$ on angle less than $\pi / 2$ which proves the proposition.

Now we can use the results from sections 3 and 4 to prove

Proposition 7.2. - Assume the curvature of $L_{1}$ does not change its sign and the line $\ell$ is a tangent to $L_{1}$ at an internal point. Then the function $I^{\prime \prime}(h)$ has exactly one simple zero in $\left(-h_{c},-h_{s}\right)$.

Proof. - First we recall that $M^{\prime \prime}(h)$, being the derivative of the period function, is nonvanishing [8]. Denote for shortness $\zeta(h)=I^{\prime \prime}(h) / M^{\prime \prime}(h)$. The phase curve $(h, \zeta(h))$ of system (2.9) is the separatrix connecting the saddle $\left(-h_{c}, w_{0}^{+}\left(-h_{c}\right)\right)$ and the node $\left(-h_{s}, w_{0}^{+}\left(-h_{s}\right)\right)$, which is verified by direct computations. Lemma 3.3 (i) yields that $\zeta(h)$ has in $\left[-h_{c},-h_{s}\right]$ either exactly one simple zero or at least 3 zeros. The latter possibility is eliminated by Corollary 4.10 as the curve $(h, \zeta(h))$ cannot have in the strip $-h_{c}<h<-h_{s}, w \geq 0$ a common point with the zero isocline $\Gamma_{0}$.

Corollary 7.3. - Under the assumptions of Proposition 7.2, the integral $I(h)$ has no zeros of multiplicity four in $\left(-h_{c},-h_{s}\right)$.

Now we are able to give the proof of Theorem 1.2: the curvature of the centroid curve $L_{1}$ at each point is non-zero. First we recall that in view of Theorem 1.1 the curvature of $L_{1}$ near its endpoints is always non-zero and of the same sign. Choose the coordinate system in which the Hamiltonian $H$ has a normal form (0.3). Then Theorem 6.2 yields that for $\mu$ close to 1 the centroid curve has a non-zero curvature at each point. Let us suppose that there exists a value $\mu_{0} \in(0,1)$ such that:

a) the curvature of $L_{1}$ is nowhere zero if $\mu \in\left(\mu_{0}, 1\right)$;

b) for $\mu=\mu_{0}$ the curvature of $L_{1}$ at some point $P_{0}=$ $\left(\xi\left(h_{0}\right), \eta\left(h_{0}\right)\right), h_{0} \in\left(-h_{c},-h_{s}\right)$ is zero: $\kappa\left(h_{0}\right)=0$.

Then for $\mu=\mu_{0}$ the curvature $\kappa(h)$ does not change the sign and moreover $\kappa$ has at least a double zero at $h_{0}$. Now we choose a perturbation $f, g$ in $(0.1)$ so that the line $\ell$, on which the divergence vanishes, will be tangential to $L_{1}$ at the point $P_{0}$. Using the regularity of $L_{1}$ and Corollary 2.1 from [16], we conclude that the integral $I(h)$ has at least a quadruple zero at $h=h_{0}$, which contradicts Corollary 7.3. Hence assertion a) above holds with $\mu_{0}=0$, thus proving the theorem. 
Proposition 7.1 and Theorem 1.2 immediately yield

COROLlaRY 7.4. - Each line can intersect $L_{1}$ in at most two points (counting the multiplicity).

Indeed, the same is true for $L_{2}$.

Now we wish to prove that each line can intersect $\mathcal{L}=L_{1} \cup L_{2}$ in at most two points. We let $\ell_{s}^{1}$ and $\ell_{c}^{1}$ denote the tangent lines to $L_{1}$ at its endpoints $Z_{1}$ and $C_{1}$ respectively. Recall, that $C_{1}$ is the centre inside the loop, $Z_{1}$ is the centroid point of the loop area and that the tangent $\ell_{s}^{1}$ passes also through the saddle $S_{1}$. Using Proposition 7.1 and Theorem 1.2 we see that $\ell_{s}^{1}$ and $\ell_{c}^{1}$ do intersect at a point $O_{1}$ placed from the convex side of $L_{1}$. Denote by $R_{1}$ the sector formed by $\ell_{s}^{1}$ and $\ell_{c}^{1}$ where $L_{1}$ lies and by $R_{1}^{o p}$ the opposite sector. The same construction applies to the second centroid curve $L_{2}$. Our goal will be achieved if we prove that $L_{1} \subset R_{2}^{o p}$ and $L_{2} \subset R_{1}^{o p}$ (see Fig. 8). For this we need the equations of the tangents at the endpoints of $L_{1}, L_{2}$. Unfortunately it is extremely difficult to obtain the equations of $\ell_{s}^{1}$ and $\ell_{s}^{2}$. For this reason we use in the above construction instead of the tangent $\ell_{s}^{1}$ the line $m^{1}=m$ from the proof of Lemma 3.1. When $H$ is given by (0.3), the lines $\ell_{c}^{1}$ and $m^{1}$ have equations (3.4) and intersect at a point $O_{1}$ placed in the fourth quadrant. Moreover, $\ell_{c}^{1}$ and $\ell_{c}^{2}$ are symmetric with respect to the origin; the same is true for $m^{1}$ and $m^{2}$. Then the desired result follows from the fact that the slope of each of these four lines is positive.

As the result just proved is in fact the most essential result of the present paper, we formulate it explicitly:

THEOREM 7.5. - Each line can intersect $\mathcal{L}=L_{1} \cup L_{2}$ in at most two points (counting the multiplicity).

With the result of Theorem 7.5 in hands, we prove our main Theorem 1, repeating the proof from [16]. In fact in order to determine the number of the limit cycles which tend to the centres or to the saddle-loops as $\varepsilon \rightarrow 0$ we apply results respectively from [5] and [24], [15], while the number of the cycles which tend to periodic orbits is given by the number of internal zeros of the Abelian integral $I(h)$. More precisely, we can summarize the content of Theorems 2.1, 2.2 and 2.3 from [16] as follows:

THEOREM 7.6. - Suppose that $H$ is a generic cubic Hamiltonian and the centroid curve $L$ is regular. Then the following statements hold:

(i) The number of zeros of the integral $I(h)$ in $\left(h_{s}, h_{c}\right)$ is equal to the number of internal intersection points between $L$ and $\ell$ (counting the multiplicities); 


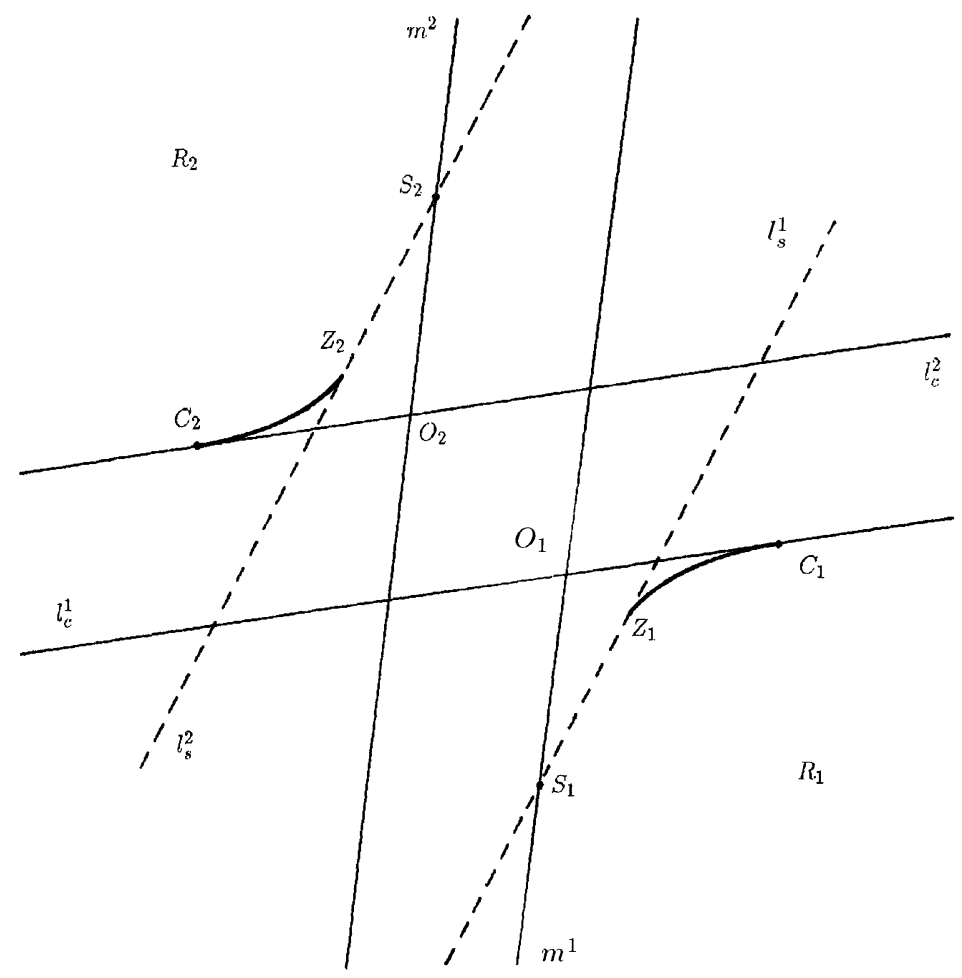

Fig. 8 .

(ii) The number of zero coefficients in the expansion

$$
I(h)=f_{1}\left(h-h_{c}\right)+f_{2}\left(h-h_{c}\right)^{2}+f_{3}\left(h-h_{c}\right)^{3}+\ldots
$$

of $I(h)$ near the critical level $h=h_{c}$ corresponding to the center $C$ is equal to the multiplicity of the intersection between $L$ and $\ell$ at $C$;

(iii) The number of zero coefficients in the expansion

$$
I(h)=c_{1}+c_{2}\left(h-h_{s}\right) \ln \left(h-h_{s}\right)+c_{3}\left(h-h_{s}\right)+\ldots
$$

of $I(h)$ near the critical level $h=h_{s}$ corresponding to the saddle loop is equal to the multiplicity of the intersection between $L$ and $\ell$ at the centroid point $Z$ of the loop area.

On the other hand, it is well known that 
(i) The number $n_{i}$ of the zeros of $I(h)$ in $\left(h_{s}, h_{c}\right)$ equals to the number of the limit cycles in (0.1) produced from the periodic annulus and tending to the corresponding ovals $H=h$ as $\varepsilon \rightarrow 0$.

(ii) The number $n_{c}$ of the zero coefficients $f_{i}, i \leq n_{c}$ in the expansion of $I(h)$ near $h=h_{c}$ is equal to the number of the limit cycles in $(0.1)$ which for $\varepsilon \rightarrow 0$ tend to the centre $C$.

(iii) The number $n_{s}$ of the zero coefficients $c_{i}, i \leq n_{s}$ in the expansion of $I(h)$ near $h=h_{s}$ is equal to the number of the limit cycles in (1.1) which tend to the saddle-loop as $\varepsilon \rightarrow 0$ (see Roussarie [24]).

Since both of the centroid curves are strictly convex and by Theorem 7.5 the number $n$ of intersection points between $\ell$ and $\mathcal{L}$ satisfies $n=n_{i}+n_{c}+n_{s} \leq 2$, Theorem 7.6 yields a bound 2 for the number of the limit cycles in (0.1). Theorem 1 is proved.

\section{ACKNOWLEDGEMENTS}

We are thankful to the referee for his critical remarks which helped us to improve the text.

\section{REFERENCES}

[1] N. A'CAMPO, Le groupe de monodromie du déploiement des singularités isolées de courbes planes I, II. I: Math. Annalen, Vol. 213, 1975, No. 1, pp. 1-32; II: In Proc. Int. Congr. Math., (Vancouver, 1974), Vol. 1, 1975, pp. 395-404.

[2] V. I. ARNOL'D, Geometrical methods in the theory of ordinary differential equations, Springer Verlag, Berlin, Heidelberg, New York, 1988.

[3] V. I. ARNOL'D, A. N. VARChENKo and S. M. GuSEIN-ZADE, Singularities of differentiable maps II, Birkhäuser Verlag, Basel, 1988.

[4] R. BAMÓN, A class of planar quadratic vector fields with a limit cycle surrounded by a saddle loop, Proceedings of the AMS, Vol. 288, 1983, pp. 719-724.

[5] N. N. Bautin, On the number of limit cycles which appear with the variation of the coefficients from an equilibrium position of focus or center type, Amer. Math. Soc. Transl., Vol. 100, 1954, pp. 1-19.

[6] R. I. BogDANOv, Bifurcation of the limit cycle of a family of planar vector fields, Selecta Math. Soviet., Vol. 1, 1981, pp. 373-387, Russian original Trudy Sem. I. G. Petrovskogo, 1976.

[7] W. A. COPPEL, A survey of quadratic systems, J. Differential Equations, Vol. 2, 1966, pp. 293-304.

[8] W. A. COPPEL and L. GAVRILOv, The period function of a Hamiltonian quadratic system, Diff. Int. Equations, Vol. 6, 1993, No. 6, pp. 1357-1365.

[9] B. DRACHMAN, S. A. VAN GILS and ZHANG ZHI-FEN, Abelian integrals for quadratic vector fields, J. reine angew. Math., Vol. 382, 1987, pp. 165-180.

[10] W. F. FREIBERGER (Editor), The International Dictionary of Applied Mathematics, D. van Nostrand Company Inc., Princeton, New Jersey, 1960.

Vol. 13, $\mathrm{n}^{\circ} 1-1996$. 
[11] L. GAVRILOV and E. HOROzOV, Limit cycles and zeroes of Abelian integrals satisfying third order Picard-Fuchs equations, In "Bifurcations of Planar Vector Fields", Lect. Notes in Math., 1455, J.-P. Françoise, R. Roussarie, eds., pp 160-186.

[12] L. GAVRILOV and E. Horozov, Limit cycles of perturbations of quadratic Hamiltonian vector fields, J. Math. Pures Appl., Vol. 72, 1993, pp. 213-238.

[13] S. M. GUSEIN-ZADE, Intersection matrices for certain singularities of functions of two variables, Funct. Anal. Appl., Vol. 8, 1974, pp. 10-13.

[14] S. M. GuSEIN-ZADE, Dynkin diagrams for singularities of functions of two variables, Funct. Anal. Appl., Vol. 8, 1974, pp. 295-300.

[15] E. HoROZOV and I. D. ILIEv, On saddle-loop bifurcations of limit cycles in perturbations of quadratic Hamiltonian systems, J. Differential Equations, Vol. 113, 1994, No. 1, pp. 84-105.

[16] E. Horozov and I. D. ILIEV, On the number of limit cycles in perturbations of quadratic Hamiltonian systems, Proc. Lond. Math. Soc., Vol. 69, 1994, No. 1, pp. 198-224.

[17] E. HoROzOV and I. D. ILIEV, Hilbert-Arnold problem for cubic Hamiltonians and limit cycles, In: Proc. Fourth Intern. Coll. Diff. Eqs, VSP Intern. Sci. Publs, Utrecht, 1994, pp. 115-124.

[18] YU. S. IL'YASHENKO, Number of zeros of certain Abelian integrals in a real domain, Funct. Anal. Appl., Vol. 11, 1978, pp. 78-79.

[19] YU. S. IL'YASHENKo, S. YAKOVENKO, Double exponential estimate for the number of zeros of complete Abelian integrals, Preprint, June 1994.

[20] A. KHOVANSKIII, Real analytic manifolds with finiteness properties and complex Abelian integrals, Funct. Anal. Appl., Vol. 18, 1984, pp. 119-128.

[21] LI JiBIN and OU YUEHUA, Global bifurcations and chaotic behaviour in a disturbed quadratic system with two centers (in Chinese), Acta Math. Appl. Sinica, Vol. 11, 1988, No. 3, pp. 312-323.

[22] G. S. Petrov, Nonoscillations of elliptic integrals, Funct. Anal. Appl., Vol. 24, 1990, No. 3, pp. 205-210.

[23] L. S. PontrJagin, Über Autoschwingungssysteme, die den Hamiltonischen nahe liegen, Zeitr. der Sowjetunion, Vol. 6, 1934, pp. 25-28.

[24] R. ROUSSARIE, On the number of limit cycles which appear by perturbation of separatrix loop of planar vector fields, Bol. Soc. Bras. Mat., Vol. 617, 1986, pp. 67-101.

[25] A. N. VARChENKo, Estimate of the number of zeros of Abelian integrals depending on parameters and limit cycles, Funct. Anal. Appl., Vol. 18, 1984, pp. 98-108.

[26] YE YAN-QIAN et al., Theory of limit cycles, Translation of Mathematical Monographs, Vol. 66, AMS, Providence, 1986.

[27] ZHI-FEN ZHANG and CHENGZHI LI, On the number of limit cycles of a class of quadratic Hamiltonian systems under quadratic perturbations, Res. Rept Peking Univ., No. 33, 1993.

[28] H. ŻOŁADEK, Quadratic systems with center and their perturbations, J. Differential Equations, Vol. 109, 1994, No. 2, pp. 223-273.

[29] A. B. GivenTAL, Sturm's theorem for hyperelliptic integrals, Leningrad Math. J., Vol. 1, 1990.

(Manuscript received March 18, 1994;

Revised version received December 28, 1994.) 